JOURNAL OF THE AMERICAN MATHEMATICAL SOCIETY

Volume 19, Number 3, Pages 527-550

S 0894-0347(06)00515-7

Article electronically published on January 19, 2006

\title{
INTEGRAL POINTS ON ELLIPTIC CURVES AND 3-TORSION IN CLASS GROUPS
}

\author{
H. A. HELFGOTT AND A. VENKATESH
}

\section{INTRODUCTION}

We give new bounds for the number of integral points on elliptic curves. The method may be said to interpolate between approaches via diophantine techniques ( $\mathrm{BP}, \mathrm{HBR}]$ ) and methods based on quasi-orthogonality in the Mordell-Weil lattice ([Sil6], GS], $\mathrm{He}$ ). We apply our results to break previous bounds on the number of elliptic curves of given conductor and the size of the 3-torsion part of the class group of a quadratic field. The same ideas can be used to count rational points on curves of higher genus.

1.1. Conductors and class groups. Let $N$ be a positive integer. We show that there are at most $O\left(N^{0.22377 \ldots}\right)$ elliptic curves over $\mathbb{Q}$ of conductor $N$. We also prove that, for every non-zero integer $D$, at most $O\left(|D|^{0.44178 \cdots}\right)$ elements of the class group of $\mathbb{Q}(\sqrt{D})$ are 3 -torsion. The latter result provides the first improvement on the trivial bound of $O\left(D^{1 / 2+\epsilon}\right)$, whereas the former improves on $O\left(N^{1 / 2+\epsilon}\right)$, which follows from the mentioned trivial bound ([BS, Theorem 1]). The new bound on 3 -torsion implies that there are at most $O\left(|D|^{0.44178 \ldots}\right)$ cubic extensions of $\mathbb{Q}$ with discriminant $D$ (see [Ha, Satz 7]). These results are derived from a new method of obtaining bounds for the number of integral (or rational) points on curves of non-zero genus.

These questions have attracted considerable interest; see, e.g., $\mathrm{Du}$. A number of authors have given improved bounds either conditionally (Wo2 $)$ or in the average ([DK], $\mathrm{Mur},[\mathrm{So}])$. The problems are intimately linked: the size of 3-torsion can be bounded above by the number of integral points of moderate height on the variety $y^{2}+D z^{2}=x^{3}$, whereas elliptic curves of given conductor correspond to $S$-integral points on a finite collection of elliptic curves of the form $y^{2}=x^{3}+C$. The question of the size of 3 -torsion is of further interest in view of its connection to the enumeration of cubic fields and to upper bounds for the ranks of elliptic curves.

The techniques in this paper are valid over an arbitrary number field. For example, one may show that the number of cubic extensions of a fixed number field $K$ with prescribed discriminant $\mathscr{I}$ is $\ll N \mathscr{I}^{1 / 2-\rho_{K}}$ for some $\rho_{K}>0$. This can be deduced without difficulty from the methods of this paper: the key result about point counting, Theorem 3.8, is stated over a number field.

Received by the editors May 21, 2004.

2000 Mathematics Subject Classification. Primary 11G05, 11R29; Secondary 14G05, 11R11.

Key words and phrases. Class groups, elliptic curves, integral points.

The second author was supported in part by NSF grant DMS-0245606.

(C)2006 American Mathematical Society Reverts to public domain 28 years from publication 
1.2. Points on curves. Let $E$ be an elliptic curve defined over a number field $K$. Let $S$ be a finite set of places of $K$. We wish to bound the cardinality of the set $E(K, S)$ of $S$-integral points on $E$.

Embed the Mordell-Weil lattice $E(K)$, modulo torsion, into $\mathbb{R}^{\operatorname{rank}(E(K))}$, so that the canonical height on $E$ is taken to the square of the Euclidean norm. Regard $E(K, S)$ as a subset of $E(K)$. One way to bound the cardinality of $E(K, S)$ is to exploit the fact that, in a certain sense, the points of $E(K, S)$ tend to be separated from each other. This idea is already present in [Sil6, GS; let us consider it in the manner of [He, §4]. After some modest slicing of $E(K, S)$, we see that any two points on the same slice are separated by almost $60^{\circ}$. We can then apply the best available results on sphere-packing $\mathrm{KL}$ to obtain a bound on the number of elements of $E(K, S)$. This bound (Corollary 3.11) improves on [GS] and seems to be the best to date. Corollary 3.12 improves on a bound of W. Schmidt Schm.

A major weakness of the relatively naive method discussed thus far is that it is very sensitive to the rank of the Mordell-Weil lattice. We have used bounds for sphere-packing problems, and such bounds typically depend exponentially on the dimension of the ambient space. This makes it difficult to apply to many natural problems, including that of 3-torsion in quadratic class groups, where one has a relatively poor bound on the rank of the Mordell-Weil lattice. In general, this problem will be particularly severe when one is bounding the number of points on $E(K, S)$ below a certain height $h_{0}$, where $h_{0}$ is comparable to the "height of $E$," i.e., the logarithm of the largest coefficient in a Weierstrass equation of $E$.

Our key idea to overcome this obstacle is to exploit a certain feature of the geometry of high-dimensional Euclidean spaces, namely, the fact that the solutions to certain special types of packing problems depend relatively weakly on the dimension of the ambient space. More precisely, consider the question: how many vectors can one pack into the unit sphere on $\mathbb{R}^{n}$ such that the angle between any two is $\geq \theta$ ? It is not difficult to see (see remark after Proposition 3.7) that one can give an upper bound independent of $n$ when $\theta>\pi / 2$. We will exploit a related but considerably deeper feature, namely, that this phenomenon persists (in a much weakened form) when $\theta<\pi / 2$ : for $\theta=\pi / 2-\alpha$ the work of Kabatjanskii and Levenshtein gives an upper bound, for small $\alpha$, of the form $\exp \left(\alpha^{2} \log \left(\alpha^{-1}\right) n\right)$. The critical feature here is that the constant $\alpha^{2} \log \left(\alpha^{-1}\right)$ depends sublinearly on $\alpha$.

We shall exploit this feature by introducing a costly type of slicing of $E(K, S)$, which allows us to increase the angle of $60^{\circ}$ and thus lowers the bound per slice sharply; we can see the amount of slicing as a parameter to be optimized. This slicing is carried out as follows: we choose an auxiliary prime $p$ and partition $E(K, S)$ into the fibers of the reduction map $E(K, S) \rightarrow E\left(\mathbb{F}_{p}\right)$; the size of $p$ is our free parameter 1

The result obtained from $90^{\circ}+\epsilon$ is the same as what arises from $[\mathrm{BP}$, modulo the difference between the canonical and the naive height. (This is no coincidence; as we will see, the similarity between the two underlying procedures runs deep.) We then show that the results depend continuously on the angle, and that $90^{\circ}$ is a locally suboptimal choice in the interval $\left[60^{\circ}, 90^{\circ}\right]$. Thus we will be able to make a

\footnotetext{
${ }^{1}$ The fact that this type of partitioning increases the angle is an instance of a very general phenomenon: rational points on an algebraic variety repel each other more strongly if they are forced to be $p$-adically close. This is already visible for integers: if $x, y \in \mathbb{Z}$ are distinct, one has $|x-y| \geq 1$, but if $x, y$ are congruent $\bmod p$, one has $|x-y| \geq p$.
} 
better choice within the interval, thus obtaining a result better than the canonicalheight analogue of [BP], and, in general, better than the pure bounds as well. It is only thus that we are able to break the $h_{3}(D) \ll D^{1 / 2}$ barrier.

The same ideas can be applied to bounding the number of rational points (or integer points) up to a certain height on curves of higher genus. This matter is discussed further in EV], where it is shown how to improve in certain contexts on the exponent 2/d occurring in the work of Heath-Brown [HBR] and Elkies [El]. We have therefore provided in the present paper only a sketch of how to extend these methods to that case; see Section 5

1.3. Relation to other work. The techniques known up to now for bounding integral points on elliptic curves did not suffice to improve on the estimates $O\left(N^{1 / 2+\epsilon}\right)$ and $O\left(D^{1 / 2+\epsilon}\right)$. Our method, like many results in Diophantine approximation, uses the fact that integer points that are $v$-adically close tend to repel each other. One may see the same underlying idea in the works of Bombieri and Pila ([BP]) and of Heath-Brown [HBR; for a discussion of the parallels between their methods and those in the present paper, see the remark at the end of subsection 3.3

Independently and simultaneously, L. B. Pierce has proved a bound on $h_{3}(D)$ that breaks $D^{1 / 2}$. Pierce's bound is $h_{3}(D) \ll D^{27 / 56+\epsilon}$, in general; for $D$ with certain divisibility properties the bound improves to $h_{3}(D) \ll D^{5 / 12+\epsilon}$. The methods in $[\mathrm{Pi}$ are quite different from those in the present paper; they are based on the square sieve.

\section{NotATION AND PRELIMINARIES}

2.1. Number fields and their places. Let $K$ be a number field. We write $\mathscr{O}_{K}$ for the ring of integers of $K, I_{K}$ for the semigroup of ideals of $\mathscr{O}_{K}, \operatorname{Cl}\left(\mathscr{O}_{K}\right)$ for the class group of $K, M_{K}$ for the set of all places of $K$, and $M_{K, \infty}$ for the set of all infinite places of $K$. We write $N_{K / \mathbb{Q}} \mathfrak{a}$ for the norm of an ideal $\mathfrak{a} \in I_{K}$. By a prime we will mean either a finite place of $K$ or the prime ideal corresponding thereto.

Let $v$ be a non-archimedean place of $K, K_{v}$ the completion of $K$ at $v$, and $p$ the prime of $\mathbb{Q}$ below $v$. We denote by $v(x): K_{v}^{*} \rightarrow \mathbb{Z}$ the valuation, normalized as usual to be surjective, and we shall normalize the absolute value $|\cdot|_{v}: K_{v}^{*} \rightarrow \mathbb{R}$ so that it extends the usual value $|\cdot|_{p}$ of $\mathbb{Q}$. Thus, for $x \in K_{v}^{*},|x|_{v}=p^{-v(x) / e_{v}}$, where $e_{v}$ is the ramification degree of $K_{v}$ over $\mathbb{Q}_{p}$.

Given a set of places $S \subset M_{K}$, we write $\mathscr{O}_{K, S}$ for the ring of $S$-integers. An $S$-integer is an $x \in K$ with $v(x) \geq 0$ for $v \notin S \cup M_{K, \infty}$. We write $M(S)$ for the product of all finite places in $S$, seen as ideals.

By $G[l]$ we mean the $l$-torsion subgroup of a group $G$. Define $h(K)=\# \mathrm{Cl}\left(\mathscr{O}_{K}\right)$, $h_{l}(K)=\#\left(\mathrm{Cl}\left(\mathscr{O}_{K}\right) / \mathrm{Cl}\left(\mathscr{O}_{K}\right)^{l}\right)=\#\left(\mathrm{Cl}\left(\mathscr{O}_{K}\right)[l]\right)$. (By \#A we mean the cardinality of a set $A$.) The number of prime ideals dividing an ideal $\mathfrak{a} \in I_{K}$ is denoted by $\omega_{K}(\mathfrak{a})$. If $a \in \mathbb{Z}$, we may write $\omega(a)$ instead of $\omega_{K}((a))$.

Given a place $v$ of $K$, we let

$$
\gamma_{v}= \begin{cases}1 & \text { if } v \text { is infinite } \\ 0 & \text { if } v \text { is finite }\end{cases}
$$

We further set $d_{v}=\left[K_{v}: \mathbb{Q}_{p}\right]$ where $p$ is the place of $\mathbb{Q}$ below $K$; in particular, $d_{v}=2$ or 1 when $v$ is complex or real, respectively. 
If $R$ is an integral domain with quotient field $K$ and $M$ is an $R$-module, we write $\operatorname{rank}_{R}(M)$ for the dimension of $M \otimes_{R} K$ over $K$.

For every $r \in \mathbb{R}$, we define

$$
\log ^{+} r=\log (\max (r, 1)) .
$$

Given $x \in K$, we define its height

$$
h_{K}(x)=\sum_{v \in M_{K}} d_{v} \log ^{+}\left(|x|_{v}\right)
$$

and its absolute height

$$
h(x)=\frac{1}{[K: \mathbb{Q}]} h_{K}(x)
$$

2.2. Elliptic curves. Let $E$ be an elliptic curve over a number field $K$. Given a field $L \supset K$, we use $E(L)$ to denote the set of $L$-valued points of $E$. (Thus $E(\mathbb{Q}$ ) is the set of rational points of an elliptic curve defined over $\mathbb{Q}$.) We take $E$ to be given by a Weierstrass equation

$$
E: y^{2}+a_{1} x y+a_{3} y=x^{3}+a_{2} x^{2}+a_{4} x+a_{6},
$$

where $a_{1}, \ldots, a_{6} \in \mathscr{O}_{K}$. By $j(E)$ (resp. $\Delta(E)$ ) we mean the $j$-invariant (resp. discriminant) of (2.4). We write $x(P), y(P)$ for the $x$ - and $y$-coordinates of a point $P \in E(\bar{K})$ other than the origin. Given a set of places $S \subset M_{L}$, we denote by $E(L, S)$ the set of $S$-integral points, i.e., points with $S$-integral coordinates:

$$
E(L, S)=\left\{P \in E(L) \backslash\{0\}: x(P), y(P) \in \mathscr{O}_{L, S}\right\} .
$$

As usual, we write $\hat{h}$ for the canonical height on $E$, defined on all points of $E(\bar{K})$. The canonical height $\hat{h}$ is a positive definite quadratic form 22 on the abelian group $E(\bar{K})$, or, by restriction, on $E(K)$. It allows itself to be expressed as a sum of local height functions $\lambda_{v}: E\left(K_{v}\right) \rightarrow \mathbb{R}$ as follows:

$$
\hat{h}(P)=\frac{1}{[K: \mathbb{Q}]} \hat{h}_{K}(P)=\frac{1}{[K: \mathbb{Q}]} \sum_{v \in M_{K}} d_{v} \lambda_{v}(P) .
$$

Local height functions are canonically defined up to an additive constant; we follow the conventions in [La, Chapter VI], and [Sil, Chapter VI], which make local heights independent of the model. Note that $\lambda_{v}(P)=\lambda_{v}(-P)$ for every place $v$ and, in particular, $\lambda_{v}\left(P_{1}-P_{2}\right)=\lambda_{v}\left(P_{2}-P_{1}\right)$ for any place $v$ and any $P_{1}, P_{2} \in E\left(K_{v}\right)$.

We recall that an elliptic curve $E$ over a non-archimedean local field $K$ is said to have potentially good reduction if it admits a model with good reduction in some extension of $K$. We say that $E$ has potentially multiplicative reduction if it does not have potentially good reduction; this occurs precisely when the $j$-invariant of $E$ is not integral. See [Sil2, Chapter VII].

\footnotetext{
${ }^{2}$ Where "positive definite" is taken to mean "mapping non-torsion elements to positive numbers."
} 


\section{INTEGRAL POINTS ON ELLIPTIC CURVES}

3.1. Uniform quasi-orthogonality. Integral points on elliptic curves tend to repel each other; so do rational points on curves of higher genus. A classical formulation of the latter fact is due to Mumford $[\mathrm{Mu}$; the former phenomenon can be seen to surface in Sil6] and GS. In order to go further, however, we must quantify this repulsion in a fashion that is more uniform and more flexible than those available to date.

As in GS, we will use local heights. Roughly speaking, we wish to establish a result of the form $\lambda_{v}(P-Q) \geq \min \left(\lambda_{v}(P), \lambda_{v}(Q)\right)$. Although this is not quite true at places of bad reduction or at the archimedean places, it is true if we subdivide $E\left(K_{v}\right)$ into a fairly small number of slices and ask that $P, Q$ lie in the same slice; see Lemmas 3.1 3.3. One feature of these lemmas is that they provide somewhat sharper results in the region where $\lambda_{v}(P) \leq 0$ than elsewhere; this will eventually be significant in dealing with points of small (global) height. We can then prove the quasi-orthogonality result in Proposition 3.4. In words, it asserts that integral points are quite far apart from each other in the Mordell-Weil lattice, and, moreover, forcing two integral points to be congruent modulo some ideal of $\mathscr{O}_{K}$ forces them even further apart in the Mordell-Weil lattice.

It should be remarked that if one is willing to accept an extra factor of size about $(1+m)^{m}$ in Theorem [3.8, where $m$ is the number of places of potentially multiplicative reduction, the proofs that follow can be considerably simplified. In this context, note that $y^{2}=x^{3}+D$ has in fact $m=0$, so this weaker version would suffice for the applications in Section 4. Indeed, for the applications of Section 4. it is not difficult to avoid local heights completely: since we deal with the curves $y^{2}=x^{3}+D$, one may use the fact that they are all twists of $y^{2}=x^{3}+1$ to prove the required special cases of Proposition 3.4 and Theorem 3.8 in an elementary fashion (cf. [He, Lemma 4.16].

Lemma 3.1. Let $E$ be an elliptic curve over a non-archimedean local field $K_{v}$ with potentially good reduction. Let $P_{1}, P_{2} \in E\left(K_{v}\right)$ be two distinct points. Then

$$
\lambda_{v}\left(P_{1}-P_{2}\right) \geq \min \left(\lambda_{v}\left(P_{1}\right), \lambda_{v}\left(P_{2}\right)\right) .
$$

Proof. Pass to an extension $L_{w}$ of $K_{v}$ on which $E$ acquires good reduction. Choose a Weierstrass equation for $E$ over $L_{w}$ such that $v(\Delta)=0$. Then $\lambda_{v}(P)=\lambda_{w}(P)=$ $\frac{1}{2} \log ^{+}\left(|x(P)|_{w}\right)$. The statement follows therefrom by direct computation. (Alternatively, use the definition of the local height in terms of the canonical filtration.)

Lemma 3.2. Let $E$ be an elliptic curve over a non-archimedean local field $K_{v}$ with potentially multiplicative reduction. Then, for any sufficiently small $\epsilon>0$, there is a partition

$$
E\left(K_{v}\right)=W_{v, 0} \cup W_{v, 1} \cup \cdots \cup W_{v, n_{v}}, \quad n_{v} \ll|\log \epsilon|,
$$

such that for any two distinct points $P_{1}, P_{2} \in W_{v, 0}$,

$$
\lambda\left(P_{1}-P_{2}\right) \geq \min \left(\lambda\left(P_{1}\right), \lambda\left(P_{2}\right)\right) \text { and } \lambda\left(P_{1}\right), \lambda\left(P_{2}\right) \geq 0,
$$

and for any two distinct points $P_{1}, P_{2} \in W_{v, j}, 1 \leq j \leq n_{v}$,

$$
\begin{aligned}
& \lambda\left(P_{1}-P_{2}\right) \geq(1-\epsilon) \max \left(\lambda\left(P_{1}\right), \lambda\left(P_{2}\right)\right), \\
& \lambda\left(P_{1}-P_{2}\right) \geq(1-2 \epsilon) \max \left(\lambda\left(P_{1}\right), \lambda\left(P_{2}\right)\right) .
\end{aligned}
$$

The implied constant is absolute. 
Proof. The elliptic curve $E$ is isomorphic, over an algebraic closure $\overline{K_{v}}$, to a Tate curve $E_{q}$ for some $q \in K_{v}^{*}$ satisfying $v(q)=-v(j)$, where $j=j(E)$ is the $j$-invariant of $E$; see [Sil, Chapter V].

There is a natural composition

$$
\beta_{v}: E\left(K_{v}\right) \rightarrow E\left(L_{w}\right) \rightarrow E\left(L_{w}\right) / E_{0}\left(L_{w}\right) \stackrel{\alpha}{\rightarrow} \mathbb{R} / \mathbb{Z} \rightarrow[0,1),
$$

where $L_{w} / K_{v}$ is the minimal extension such that $E$ acquires split multiplicative reduction over $L_{w}$, and the map $\alpha$ is given by $\alpha(t)=v(t) / v(q)$ on the Tate curve. (See [La, pp. 68-69]; cf. [GS, p. 270].) For every $P \in E\left(L_{w}\right)$,

$$
\lambda(P)=-\frac{1}{2} B_{2}\left(\beta_{v}(P)\right) \log |q|_{w}-\iota(P) \log |\pi|_{w},
$$

where $w$ is an extension of $v$ as above, $\pi$ is a uniformizer of $w, B_{2}(t)=t^{2}-t+1 / 6$, $\iota(P)=0$ when $P \notin E_{0}\left(L_{w}\right)$, and, if $P \in E_{0}\left(L_{w}\right), \iota(P)$ is the largest integer $\iota$ such that $P \in E_{\iota}\left(L_{w}\right)$, where

$$
E\left(L_{w}\right) \supset E_{0}\left(L_{w}\right) \supset E_{1}\left(L_{w}\right) \supset \cdots
$$

is the canonical filtration of $E\left(L_{w}\right)$. (See [La, pp. 68-70]; cf. [GS, p. 270].) Clearly

$$
\iota\left(P_{1}-P_{2}\right) \geq \min \left(\iota\left(P_{1}\right), \iota\left(P_{2}\right)\right) .
$$

If $\beta_{v}\left(P_{1}\right)=\beta_{v}\left(P_{2}\right)=0$, it follows that $\lambda\left(P_{1}-P_{2}\right) \geq \min \left(\lambda\left(P_{1}\right), \lambda\left(P_{2}\right)\right)$. Define, then, $W_{v, 0}=\left\{P \in E\left(K_{v}\right): \beta_{v}(P)=0\right\}$. It remains to partition $\left\{P \in E\left(K_{v}\right)\right.$ : $\left.\beta_{v}(P) \neq 0\right\}$. Partition $(0,1 / 2]$ into sets $U_{0}, U_{1}, \ldots, U_{m}$, where $m=\left\lceil\log _{3 / 2}(6 / \epsilon)\right\rceil$ :

$$
\begin{aligned}
& U_{0}=(0, \epsilon / 12], U_{m}=\left((3 / 2)^{m-1} \epsilon / 12,1 / 2\right], \\
& U_{j}=\left((3 / 2)^{j-1} \epsilon / 12,(3 / 2)^{j} \epsilon / 12\right], \quad 1 \leq j<m .
\end{aligned}
$$

Note that $B_{2}(t)$ is decreasing on $t \in[0,1 / 2]$. Suppose $t_{1}, t_{2}$ both belong to $U_{j}$ and $t_{1} \geq t_{2}$. If $j=0$, we have

$$
\begin{aligned}
B_{2}\left(t_{1}-t_{2}\right) \geq B_{2}(\epsilon / 12) \geq 1 / 6-\epsilon / 12 & =(1-\epsilon) B_{2}(0)+\epsilon / 12 \\
> & (1-2 \epsilon) B_{2}(0)+\epsilon / 12 .
\end{aligned}
$$

If $j \geq 1$, then $u \leq t_{2} \leq t_{1} \leq 3 u / 2$, where $u=(3 / 2)^{j-1} \epsilon / 12$. Then

$$
\begin{gathered}
B_{2}\left(t_{1}-t_{2}\right) \geq B_{2}(u / 2), \\
B_{2}(u / 2) \geq(1-\epsilon) B_{2}(u)+\epsilon / 12, \quad B_{2}(u / 2) \geq(1-2 \epsilon) B_{2}(u)+\epsilon / 12 .
\end{gathered}
$$

(The last two inequalities are proved in two cases according to whether $B_{2}(u) \geq$ $1 / 12$ or $B_{2}(u)<1 / 12$. In the former case we have $B_{2}(u / 2) \geq B_{2}(u)=(1-\epsilon) B_{2}(u)+$ $\epsilon B_{2}(u) \geq(1-\epsilon) B_{2}(u)+\epsilon / 12>(1-2 \epsilon) B_{2}(u)+\epsilon / 12$. In the latter case, $u>1 / 11$ and $B_{2}(u / 2)-B_{2}(u)>1 / 30$; in particular $B_{2}(u / 2)-(1-\epsilon) B_{2}(u)-\epsilon / 12 \geq 1 / 30+$ $\epsilon B_{2}(u)-\epsilon / 12$. Since $B_{2}(u) \geq-1 / 12$, it follows that $B_{2}(u / 2)-(1-\epsilon) B_{2}(u)-\epsilon / 12 \geq$ $1 / 30-\epsilon / 6 \geq 0$, where we assume $\epsilon<1 / 5$; similarly, $B_{2}(u / 2)-(1-2 \epsilon) B_{2}(u)-\epsilon / 12 \geq$ $1 / 30-\epsilon / 4 \geq 0$, where we assume $\epsilon<2 / 15$.)

Combining (3.2) and (3.3) we obtain 3

$$
\begin{aligned}
& B_{2}\left(t_{1}-t_{2}\right) \geq(1-\epsilon) \max _{j=1,2} B_{2}\left(t_{j}\right)+\epsilon / 12, \\
& B_{2}\left(t_{1}-t_{2}\right) \geq(1-2 \epsilon) \max _{j=1,2} B_{2}\left(t_{j}\right)+\epsilon / 12
\end{aligned}
$$

\footnotetext{
${ }^{3}$ The term $\epsilon / 12$ in the displayed equation will be used in the proof of Lemma 3.3
} 
for all $t_{1}, t_{2} \in U_{j}, 0 \leq j \leq m$, where $t_{1} \geq t_{2}$. Define

$$
\begin{aligned}
& W_{v, 2 j+1}=\left\{P \in E\left(K_{v}\right): \beta_{v}(P) \in U_{j}\right\}, \\
& W_{v, 2 j+2}=\left\{P \in E\left(K_{v}\right): \beta_{v}(-P) \in U_{j}, \beta_{v}(P) \neq 1 / 2\right\}
\end{aligned}
$$

for $0 \leq j \leq m$. We set $n_{v}=2 m+2$ and we are done.

Lemma 3.3. Let $E$ be an elliptic curve over $\mathbb{C}$. Then, for any sufficiently small $\epsilon>0$, there is a partition

$$
E(\mathbb{C})=W_{0} \cup W_{1} \cup \cdots \cup W_{n}, \quad n \ll \epsilon^{-2}|\log \epsilon|^{2},
$$

such that for any two distinct points $P_{1}, P_{2} \in W_{j}, 0 \leq j \leq 5$,

$$
\lambda\left(P_{1}-P_{2}\right) \geq(1-\epsilon) \min \left(\lambda\left(P_{1}\right), \lambda\left(P_{2}\right)\right) \text { and } \lambda\left(P_{1}\right), \lambda\left(P_{2}\right) \geq 0,
$$

and for any two distinct points $P_{1}, P_{2} \in W_{j}, 6 \leq j \leq n$,

$$
\begin{aligned}
& \lambda\left(P_{1}-P_{2}\right) \geq(1-\epsilon) \max \left(\lambda\left(P_{1}\right), \lambda\left(P_{2}\right)\right), \\
& \lambda\left(P_{1}-P_{2}\right) \geq(1-2 \epsilon) \max \left(\lambda\left(P_{1}\right), \lambda\left(P_{2}\right)\right) .
\end{aligned}
$$

The implied constant in (3.5) is absolute.

Proof. There is an isomorphism $E(\mathbb{C}) \stackrel{u}{\rightarrow} \mathbb{C} /(\mathbb{Z}+\tau \mathbb{Z})$ for some $\tau$ in the usual fundamental domain of $\mathrm{SL}_{2}(\mathbb{Z}) \backslash \mathbb{H}$. Note especially that $\Im(\tau) \geq \sqrt{3} / 2$. Write $u(P)=u_{P, 1}+\tau u_{P, 2}, u_{P, 1}, u_{P, 2} \in\left(-\frac{1}{2}, \frac{1}{2}\right]$. Define $q=e^{2 \pi i \tau}, q_{u}(P)=e^{2 \pi i u_{P}}$-note that $|q| \leq e^{-\pi \sqrt{3}}$. The local height is given by

$$
\lambda(P)=-\frac{1}{2} B_{2}\left(u_{P, 2}\right) \log |q|-\log \left|g_{0}\left(q_{u}(P)\right)\right|,
$$

where

$$
g_{0}(t)=(t-1) \prod_{n=1}^{\infty}\left(1-q^{n} t\right)\left(1-q^{n} t^{-1}\right)
$$

and $B_{2}(t)$ is as in the proof of Lemma 3.2. (See, e.g. Sil, Chapter VI, Theorem 3.4]) We partition $[0,1 / 2]$ into sets $U_{0}, U_{1}, \ldots, U_{m}$ as in the same proof. For the present proof we adjoin 0 to $U_{0}$, since Lemma 3.2 partitions only $(0,1 / 2)$.

Whenever $t_{1}, t_{2}$, with $t_{2} \leq t_{1}$, belong to the same set $U_{j}$, one obtains

$$
\begin{aligned}
& B_{2}\left(t_{1}-t_{2}\right) \geq(1-\epsilon) \max _{j=1,2} B_{2}\left(t_{j}\right)+\epsilon / 12, \\
& B_{2}\left(t_{1}-t_{2}\right) \geq(1-2 \epsilon) \max _{j=1,2} B_{2}\left(t_{j}\right)+\epsilon / 12
\end{aligned}
$$

as in (3.4), where we assume $\epsilon<2 / 15$.

Let $P_{1}, P_{2} \in E(\mathbb{C})$. Since $\lambda(P)=\lambda(-P)$, we may assume $0 \leq u_{P_{2}, 2} \leq u_{P_{1}, 2} \leq$ $1 / 2$ without loss of generality. Let $\mathscr{A}$ be the annulus $\left\{z:|q|^{1 / 2} \leq|z| \leq 1\right\}$; thus whenever $0 \leq u_{P, 2} \leq 1 / 2$, we have $q_{u}(P) \in \mathscr{A}$.

In view of (3.10) and $-\frac{1}{2} \log |q| \geq \frac{\pi \sqrt{3}}{2}>2$, it will suffice (see (3.22) $)$ to partition $\mathscr{A}$ into sets $V_{0}, V_{1}, \ldots, V_{m^{\prime}}$ such that

$$
\begin{aligned}
-\log \left|g_{0}\left(q_{u}\left(P_{1}-P_{2}\right)\right)\right| & \geq(1-\epsilon) \min _{j=1,2}\left(-\log \left|g_{0}\left(q_{u}\left(P_{j}\right)\right)\right|\right)-\epsilon / 6, \\
0 \leq u_{P_{2}, 2}, u_{P_{1}, 2} & \leq \epsilon / 12, \quad \lambda\left(P_{1}\right), \lambda\left(P_{2}\right) \geq 0
\end{aligned}
$$


if $u_{P_{2}, 2} \leq u_{P_{1}, 2}$ and $q_{u}\left(P_{1}\right), q_{u}\left(P_{2}\right) \in V_{j}$ for some $j \in\{0,1,2\}$, and

$$
\begin{aligned}
& -\log \left|g_{0}\left(q_{u}\left(P_{1}-P_{2}\right)\right)\right| \geq(1-\epsilon) \max _{j=1,2}\left(-\log \left|g_{0}\left(q_{u}\left(P_{j}\right)\right)\right|\right)-\epsilon / 6, \\
& -\log \left|g_{0}\left(q_{u}\left(P_{1}-P_{2}\right)\right)\right| \geq(1-2 \epsilon) \max _{j=1,2}\left(-\log \left|g_{0}\left(q_{u}\left(P_{j}\right)\right)\right|\right)-\epsilon / 6
\end{aligned}
$$

if $u_{P_{2}, 2} \leq u_{P_{1}, 2}$ and $q_{u}\left(P_{1}\right), q_{u}\left(P_{2}\right) \in V_{j}$ for some $j \in\left\{3,4, \ldots, m^{\prime}\right\}$.

The region near $z=1$. Let $D_{\text {big }}=\{\delta \in \mathbb{C}:|\delta| \leq 1 / 2\}, D_{\text {small }}=\{\delta \in \mathbb{C}:|\delta| \leq$ $1 / 8\}$. Note $\left(1+D_{\text {small }}\right) \cdot\left(1+D_{\text {small }}\right)^{-1} \subset 1+D_{\text {big }}$.

For $t=1+\delta, \delta \in D_{\mathrm{big}}$,

$$
\begin{aligned}
-\log \left|g_{0}(t)\right| & =-\log |t-1|-\sum_{n=1}^{\infty} \log \left|1-q^{n}\left(t+t^{-1}\right)+q^{2 n}\right| \\
& =-\log |\delta|-2 \sum_{n=1}^{\infty} \log \left|1-q^{n}\right|+O\left(|\delta|^{2}\right) .
\end{aligned}
$$

(Here we use the fact that $|q| \leq e^{-\pi \sqrt{3}}$.) Thus, for any $\delta_{1}, \delta_{2} \in D_{\text {small }}$ with $\arg \left(\delta_{1} / \delta_{2}\right) \in\left[-\frac{\pi}{3}, \frac{\pi}{3}\right]$,

$$
\begin{aligned}
-\log \left|g_{0}\left(\frac{1+\delta_{1}}{1+\delta_{2}}\right)\right| & =-\log \left|\delta_{1}-\delta_{2}\right|-2 \sum_{n=1}^{\infty} \log \left|1-q^{n}\right|+O\left(\max \left(\left|\delta_{1}\right|,\left|\delta_{2}\right|\right)\right) \\
& \geq \min _{j=1,2}\left(-\log \left|g_{0}\left(1+\delta_{j}\right)\right|\right)+O\left(\max _{j=1,2}\left|\delta_{j}\right|\right) .
\end{aligned}
$$

We can thus define the sets

$$
V_{k}=\left\{z \in \mathscr{A}:|1-z| \leq \kappa_{0} \epsilon, \arg (1-z) \in\left[-\frac{\pi}{2}+\frac{\pi}{3} k,-\frac{\pi}{2}+\frac{\pi}{3}(k+1)\right]\right\},
$$

where $k=0,1,2$ and $\kappa_{0}$ is small enough so that (a) $O\left(\max _{j=1,2}\left|\delta_{j}\right|\right)$ in (3.14) is less than $\epsilon / 6$ in absolute value when $\left|\delta_{j}\right| \leq \kappa_{0} \epsilon$, (b) $|\log | 1-\kappa_{0} \epsilon|| /(\pi \sqrt{3}) \leq \epsilon / 12$, and (c) $-\log \left|g_{0}(1+\delta)\right| \geq 0$, for any $q$, whenever $|\delta| \leq \kappa_{0} \epsilon$. The conditions in (3.11) are then satisfied.

The region near $z=0$. We will partition the region $\{z \in \mathscr{A}:|z| \leq \kappa \epsilon\}$ for some constant $\kappa$.

For $t \in \mathscr{A}$ we have the bounds

$$
\begin{aligned}
\prod_{n=1}^{\infty}\left(1-|q|^{n-1 / 2}-|q|^{n+1 / 2}-|q|^{2 n}\right) & \leq\left|\prod_{n=1}^{\infty}\left(1-q^{n} t\right)\left(1-q^{n} t^{-1}\right)\right|, \\
\left|\prod_{n=1}^{\infty}\left(1-q^{n} t\right)\left(1-q^{n} t^{-1}\right)\right| & \leq \prod_{n=1}^{\infty}\left(1+|q|^{n+1 / 2}\right)\left(1+|q|^{n-1 / 2}\right) .
\end{aligned}
$$

In particular, there is an absolute constant $\kappa_{1}$ such that, if $t \in \mathscr{A}$ and $|q|^{1 / 2} \leq \kappa_{1} \epsilon$,

$$
e^{-\epsilon / 18} \leq\left|\prod_{n=1}^{\infty}\left(1-q^{n} t\right)\left(1-q^{n} t^{-1}\right)\right| \leq e^{\epsilon / 18} .
$$

We will eventually choose $\kappa \leq \kappa_{1}$, so that if $|q|^{1 / 2}>\kappa_{1} \epsilon$, then $|z|>\kappa_{1} \epsilon$ for all $z \in \mathscr{A}$ and the set $\{t \in \mathscr{A}:|t|<\kappa \epsilon\}$ is empty. We may therefore assume that 
INTEGRAL POINTS ON ELLIPTIC CURVES AND 3-TORSION IN CLASS GROUPS 535 $|q|^{1 / 2} \leq \kappa_{1} \epsilon$ and that (3.16) holds. Now, for any $t \in \mathscr{A}$ such that $e^{-\epsilon / 18} \leq|t-1| \leq$ $e^{\epsilon / 18}$,

$$
|-\log | g_{0}(t)||=|-\log | t-1|-\log | \prod_{n=1}^{\infty}\left(1-q^{n} t\right)\left(1-q^{n} t^{-1}\right)|| \leq \epsilon / 9 .
$$

For $k=1,2, \ldots, 6$, let

$$
V_{k+2}=\left\{z \in \mathscr{A}:|z| \leq \kappa_{2} \epsilon, \arg (z) \in\left[\frac{(k-1) \pi}{3}, \frac{k \pi}{3}\right)\right\}
$$

where $\kappa_{2}$ is an absolute constant such that $e^{-\epsilon / 18} \leq|z-1| \leq e^{\epsilon / 18}$ for $|z| \leq \kappa_{2} \epsilon$. Suppose $P_{1}, P_{2} \in E(\mathbb{C})$ are such that $0 \leq u_{P_{2}, 2} \leq u_{P_{1}, 2} \leq 1 / 2$ and $q_{u}\left(P_{1}\right), q_{u}\left(P_{2}\right) \in$ $V_{k+2}$ for some $1 \leq k \leq 6$. Then $q_{u}\left(P_{1}-P_{2}\right)$ belongs to $\mathscr{A}$ and satisfies

$$
\left|q_{u}\left(P_{1}-P_{2}\right)-1\right| \leq 1
$$

Then (3.16) shows that $-\log \left|g\left(q_{u}\left(P_{1}-P_{2}\right)\right)\right| \geq-\epsilon / 18$. Combining this with (3.17), we obtain

$$
\begin{aligned}
& -\log \left|g_{0}\left(q_{u}\left(P_{1}-P_{2}\right)\right)\right| \geq(1-\epsilon) \max _{j=1,2}\left(-\log \left|g_{0}\left(q_{u}\left(P_{j}\right)\right)\right|\right)-\epsilon / 6, \\
& -\log \left|g_{0}\left(q_{u}\left(P_{1}-P_{2}\right)\right)\right| \geq(1-2 \epsilon) \max _{j=1,2}\left(-\log \left|g_{0}\left(q_{u}\left(P_{j}\right)\right)\right|\right)-\epsilon / 6
\end{aligned}
$$

for $P_{1}, P_{2} \in E(\mathbb{C})$ with $u\left(P_{1}\right), u\left(P_{2}\right) \in V_{k+2}$. We set $\kappa=\min \left(\kappa_{1}, \kappa_{2}\right)$ and we are done.

The remaining region. It remains to partition the region $R=\{z \in \mathscr{A}:|z|>$ $\left.\kappa \epsilon,|1-z|>\kappa_{0} \epsilon\right\}$.

By virtue of (3.9) and (3.15), if $q_{u}(P) \in \mathscr{A}$, then $-\log \left|g\left(q_{u}(P)\right)\right|$ differs from $-\log \left|q_{u}(P)-1\right|$ by an absolutely bounded constant. In particular, there are absolute constants $c_{1}, c_{2}, c_{3}$ such that, for any $c<1$,

$$
-\log \left|g_{0}\left(q_{u}\left(P^{\prime}\right)\right)\right| \geq-\log (c \epsilon)+c_{1}
$$

whenever $q_{u}\left(P^{\prime}\right) \in \mathscr{A},\left|q_{u}\left(P^{\prime}\right)-1\right| \leq c \epsilon$, and

$$
c_{3} \leq-\log \left|g_{0}\left(q_{u}(P)\right)\right| \leq-\log (\epsilon)+c_{2}
$$

for all $P \in R$. By (3.19) and (3.20), we may choose a sufficiently small (absolute) constant $c$ such that

$$
\begin{aligned}
& -\log \left|g_{0}\left(q_{u}\left(P^{\prime}\right)\right)\right| \geq-(1-\epsilon) \log \left|g_{0}\left(q_{u}(P)\right)\right|, \\
& -\log \left|g_{0}\left(q_{u}\left(P^{\prime}\right)\right)\right| \geq-(1-2 \epsilon) \log \left|g_{0}\left(q_{u}(P)\right)\right|
\end{aligned}
$$

for all $P, P^{\prime}$ with $q_{u}(P) \in R, q_{u}\left(P^{\prime}\right) \in \mathscr{A},\left|q_{u}\left(P^{\prime}\right)-1\right|<c \epsilon$.

Now, for any $P_{1}, P_{2}$ with $q_{u}\left(P_{1}\right), q_{u}\left(P_{2}\right) \in R, 0 \leq u_{P_{2}, 2} \leq u_{P_{1}, 2} \leq 1 / 2$ and

$$
\left|\Re \log \frac{q_{u}\left(P_{1}\right)}{q_{u}\left(P_{2}\right)}\right|,\left|\Im \log \frac{q_{u}\left(P_{1}\right)}{q_{u}\left(P_{2}\right)}\right| \leq \frac{c \epsilon}{2},
$$

we have $\left|q_{u}\left(P_{1}-P_{2}\right)-1\right|<c \epsilon$. Hence it is enough to partition $\log (R)$ into squares of side $c \epsilon / 2$. Since $\log (R)$ is contained in the rectangle $[\log (\kappa \epsilon), 0] \times[-\pi, \pi]$, there are $O\left(\epsilon^{-2}|\log \epsilon|\right)$ such squares. Their images under exp partition $R$ into sets $V_{9}, V_{10}, \ldots, V_{m^{\prime}}$, with $m^{\prime} \ll \epsilon^{-2}|\log \epsilon|$. We have $q_{u}\left(P_{1}-P_{2}\right) \in \mathscr{A},\left|q_{u}\left(P_{1}-P_{2}\right)-1\right| \leq$ 
$c \epsilon$ if $P_{1}, P_{2} \in V_{k}, 0 \leq u_{P_{2}, 2} \leq u_{P_{1}, 2} \leq 1 / 2$ for some $9 \leq k \leq m^{\prime}$. By (3.21), we may conclude that, for $P_{1}, P_{2} \in E(\mathbb{C})$ with $u\left(P_{1}\right), u\left(P_{2}\right) \in V_{k}, 9 \leq k \leq m^{\prime}$, we have

$$
\begin{aligned}
& -\log \left|g_{0}\left(q_{u}\left(P_{1}-P_{2}\right)\right)\right| \geq(1-\epsilon) \max _{j=1,2}\left(-\log \left|g_{0}\left(q_{u}\left(P_{j}\right)\right)\right|\right), \\
& -\log \left|g_{0}\left(q_{u}\left(P_{1}-P_{2}\right)\right)\right| \geq(1-2 \epsilon) \max _{j=1,2}\left(-\log \left|g_{0}\left(q_{u}\left(P_{j}\right)\right)\right|\right),
\end{aligned}
$$

which certainly imply (3.12).

Conclusion. Let $u_{2}: E(\mathbb{C}) \rightarrow(-1 / 2,1 / 2]$ be the map $P \mapsto u_{P, 2}$. We partition $E(\mathbb{C})$ into the sets

$$
u_{2}^{-1}\left(U_{i}\right) \cap q_{u}^{-1}\left(V_{k}\right), \quad u_{2}^{-1}\left(\left\{x \in-U_{i}: x \neq 0,-1 / 2\right\}\right) \cap\left(-q_{u}^{-1}\left(V_{k}\right)\right),
$$

where $0 \leq i \leq m, 0 \leq k \leq m^{\prime}$. The inequality $0 \leq u_{P_{2}, 2}, u_{P_{1}, 2} \leq \epsilon / 12$ in (3.11) ensures that $u_{2}^{-1}\left(U_{i}\right) \cap q_{u}^{-1}\left(V_{k}\right)=\emptyset$ for $k=0,1,2, i \neq 0$. We define $W_{0}, \ldots, W_{5}$ to be the sets in (3.22) arising from $0 \leq k \leq 2, i=0$, and $W_{6}, W_{7}, \ldots, W_{m}$ to be the other non-empty sets in (3.22).

Proposition 3.4. Let $E$ be an elliptic curve over a number field $K$, given by a Weierstrass equation (2.4). Let $S$ be a finite set of places of $K$, including all infinite places and all primes dividing the discriminant of $E$.

Let $P_{1}, P_{2} \in E(K, S)$ be two distinct $S$-integral points. Let $\epsilon$ be sufficiently small. Assume that $P_{1}$ and $P_{2}$ belong to the same set $W_{v, i}$ for every infinite place $v$ and every place $v$ of potentially multiplicative reduction (see (3.1), (3.5)). Furthermore, suppose that

$$
\sum_{v \in T} d_{v}\left|\lambda_{v}\left(P_{1}\right)-\lambda_{v}\left(P_{2}\right)\right| \leq \epsilon \max _{j=1,2} \sum_{v \in T} d_{v} \lambda_{v}\left(P_{j}\right),
$$

where $T=\left\{v \in S: \lambda_{v}\left(P_{1}\right) \geq 0\right.$ and $\left.\lambda_{v}\left(P_{2}\right) \geq 0\right\}$. Let $\mathscr{I}$ be an ideal of $\mathscr{O}_{K}$ not divisible by any primes in $S$. Assume that $P_{1}$ and $P_{2}$ have the same reduction modulo $\mathscr{I}$. Then

$$
\hat{h}\left(P_{1}-P_{2}\right) \geq(1-2 \epsilon) \max _{j=1,2} \hat{h}\left(P_{j}\right)+\frac{\log (N \mathscr{I})}{[K: \mathbb{Q}]} .
$$

Proof. For every finite place $v$ of good reduction, $\lambda_{v}(P) \geq 0$ (by, e.g., La, Theorem VI.4.3] or [Sil, Theorem VI.4.1]). Hence

$$
\hat{h}_{K}\left(P_{1}-P_{2}\right) \geq \sum_{v \in S} d_{v} \lambda_{v}\left(P_{1}-P_{2}\right)+\sum_{\substack{v \text { finite } \\ v(\mathscr{\mathscr { I }})>0}} d_{v} \lambda_{v}\left(P_{1}-P_{2}\right) .
$$

By Lemmas 3.1, 3.2 and 3.3, together with (3.23),

$$
\begin{aligned}
\sum_{v \in T} d_{v} \lambda_{v}\left(P_{1}-P_{2}\right) & \geq(1-\epsilon) \sum_{v \in T} d_{v} \min _{j=1,2}\left(\lambda_{v}\left(P_{j}\right)\right) \\
& \geq(1-\epsilon) \sum_{v \in T} d_{v} \max _{j=1,2}\left(\lambda_{v}\left(P_{j}\right)\right)-\epsilon \max _{j=1,2} \sum_{v \in T} d_{v} \lambda_{v}\left(P_{j}\right), \\
\sum_{v \in S-T} d_{v} \lambda_{v}\left(P_{1}-P_{2}\right) & \geq(1-2 \epsilon) \sum_{v \in S-T} d_{v} \max _{j=1,2}\left(\lambda_{v}\left(P_{j}\right)\right) .
\end{aligned}
$$

${ }^{4}$ Under the stated assumptions on $E$ and $\mathscr{I}$, there is a well-defined reduction map $E(K) \rightarrow$ $E\left(\mathscr{O}_{K} / \mathscr{I}\right)$. 
Note that $\max _{j=1,2} \sum_{v \in T} d_{v} \lambda_{v}\left(P_{j}\right) \leq \sum_{v \in T} d_{v} \max \left(\lambda_{v}\left(P_{1}\right), \lambda_{v}\left(P_{2}\right)\right)$. Thus

$$
\sum_{v \in S} d_{v} \lambda_{v}\left(P_{1}-P_{2}\right) \geq(1-2 \epsilon) \max _{j=1,2} \sum_{v \in S} d_{v} \lambda_{v}\left(P_{j}\right) .
$$

Since $x\left(P_{1}\right), x\left(P_{2}\right)$ are $S$-integers and $S$ contains all infinite places and all primes dividing the discriminant of $E$, we see that

$$
\lambda_{v}\left(P_{j}\right)=\frac{1}{2} \log ^{+}\left(\left|x\left(P_{j}\right)\right|_{v}\right)=0
$$

for $v \notin S$.

It remains to consider $\lambda_{v}\left(P_{1}-P_{2}\right)$ for $v$ finite, $v(\mathscr{I})>0$. Let $\mathfrak{p}_{v}$ be the corresponding prime ideal of $\mathscr{O}_{K}$ and $n_{v}$ its multiplicity in $\mathscr{I}$. The point $P_{1}-P_{2}$ is not $O$, but it is mapped to the origin when reduced modulo $\mathfrak{p}_{v}^{n_{v}}$. Hence $v\left(x\left(P_{1}-P_{2}\right)\right) \leq$ $-2 n_{v}$. Therefore $\lambda_{v}\left(P_{1}-P_{2}\right) \geq n_{v} e_{v}^{-1} \log \left(p_{v}\right)$, where $p_{v}$ is the rational prime lying under $v$ and $e_{v}$ is the ramification degree of $K_{v}$ over $\mathbb{Q}_{p}$. We note that $\sum_{v(\mathscr{I})>0} d_{v} n_{v} e_{v}^{-1} \log \left(p_{v}\right)=\log (N \mathscr{I})$ to conclude.

3.2. Slicing and packing. We will use Proposition 3.4 to give an upper bound on the number of $S$-integral points on the curve $E: y^{2}=x^{3}+d$. Any application of quasi-orthogonality leads fairly naturally to a bound of the form

$$
\text { \# of integer points on } E \ll C^{\operatorname{rank}(E)+\# S} \text {, }
$$

for some constant $C$ (see [GS]). However, in applications such as estimating the size of 3 -torsion, the size of $C$ is crucial; if $C$ is too large, one does not recover even the trivial bounds on 3-torsion. One may optimize the bound by applying sphere packing (cf. $[\mathrm{He}$ ); in order to make this approach particularly effective, we first partition the set of integer points on $E$ and then apply sphere-packing bounds to each part separately.

For $\vec{x}=\left(x_{i}\right)_{1 \leq i \leq n} \in \mathbb{R}^{n}$, we set $|\vec{x}|_{1}=\sum_{1 \leq i \leq n}\left|x_{i}\right|$. Let $d(\vec{x}, \vec{y})=|\vec{x}-\vec{y}|_{1}$ be the associated metric on $\mathbb{R}^{n}$. Let $\mathbb{R}_{\geq 0}=\{z \in \mathbb{R}: z \geq 0\}$.

Lemma 3.5. Let $c_{1}, c_{2}$ be positive real numbers, $0<\epsilon<1 / 2$, $n$ a non-negative integer. Let $S=\left\{\vec{x} \in \mathbb{R}_{\geq 0}^{n}: c_{1} \leq \sum_{i=1}^{n} x_{i}<c_{2}\right\}$. Then there is a subset $T \subset \mathbb{R}_{\geq 0}^{n}$ and an explicit, absolute constant $C>0$ such that

$$
\# T \leq C^{n} \epsilon^{-(n+1)}\left(1+\log \left(c_{2} / c_{1}\right)\right)
$$

and the $\ell_{1}$-balls $B\left(P, \epsilon|P|_{1}\right)$, for $P \in T$, cover all of $S$.

Proof. The idea is to slice $S$ into a union of regions where $|x|_{1}$ is almost constant and then to consider the points on a lattice in each of these regions.

Since we may replace $\epsilon$ by $\epsilon / 2$, it suffices to cover $S$ by balls $B\left(P, 2 \epsilon|P|_{1}\right)$. Let $\lfloor z\rfloor$ be the largest integer no greater than $z$. For $\vec{x}=\left(x_{i}\right)_{1 \leq i \leq n} \in \mathbb{R}^{n}$, we set $\lfloor\vec{x}\rfloor=\left(\left\lfloor x_{i}\right\rfloor\right)_{1 \leq i \leq n}$. Define $M=\frac{\log \left(c_{2} / c_{1}\right)}{\log (1+\epsilon)}$. Set

$$
T=\bigcup_{0 \leq m<M} \frac{c_{1} \epsilon(1+\epsilon)^{m}}{n}\left\{\vec{y} \in \mathbb{Z}_{\geq 0}^{n}: n\left(\epsilon^{-1}-1\right) \leq|\vec{y}|_{1}<n\left(1+\epsilon^{-1}\right)\right\} .
$$

Then $T$ has the required property: given $\vec{x} \in S$, set

$$
m(\vec{x})=\left\lfloor\frac{\log \left(|\vec{x}|_{1} / c_{1}\right)}{\log (1+\epsilon)}\right\rfloor, \quad \vec{y}(\vec{x})=\left\lfloor\frac{n \vec{x}}{\left.c_{1} \epsilon(1+\epsilon)^{m(\vec{x})}\right\rfloor .}\right.
$$


Then $P=\frac{c_{1} \epsilon(1+\epsilon)^{m(\vec{x})}}{n} \vec{y}(\vec{x})$ belongs to $T$. Moreover, $P$ satisfies $d(\vec{x}, P) \leq \frac{\epsilon}{1-\epsilon}|P|_{1} \leq$ $2 \epsilon|P|_{1}$, by virtue of the fact that $d(\vec{z},\lfloor\vec{z}\rfloor) \leq n$ for any $\vec{z} \in \mathbb{R}^{n}$. It remains to estimate $\# T$ :

$$
\begin{aligned}
\# T & \leq\left(1+\frac{\log \left(c_{2} / c_{1}\right)}{\log (1+\epsilon)}\right) \cdot \#\left\{\vec{y} \in \mathbb{Z}_{\geq 0}^{n}: n\left(\epsilon^{-1}-1\right) \leq|\vec{y}|_{1} \leq n\left(1+\epsilon^{-1}\right)\right\} \\
& \leq\left(1+\frac{\log \left(c_{2} / c_{1}\right)}{\log (1+\epsilon)}\right) \frac{\left(n\left(1+\epsilon^{-1}\right)+n\right)^{n}}{n !} .
\end{aligned}
$$

The result follows by Stirling's formula.

We will need lower bounds on the canonical height. Note that there are strong bounds for the number of points of moderately low height [Da]; such bounds could be used in place of the following proposition.

Proposition 3.6. Let $E$ be an elliptic curve over a number field $K$. There is an absolute constant $0<\kappa<1$ such that, for every non-torsion point $P \in E(K)$,

$$
\hat{h}(P)>\kappa^{m+[K: \mathbb{Q}]} \max (1, h(j)),
$$

where $m$ is the number of places of $K$ where $E$ has potentially multiplicative reduction and $j=j(E)$ is the $j$-invariant of $E$.

Proof. By the proof of the theorem in [Sil4, §4]; see also [Sil5, Theorem 7].

We shall use the remarkable bounds of Kabatjanskii and Levenshtein.

Proposition 3.7. Let $A(n, \theta)$ be the maximal number of points that can be arranged on the unit sphere of $\mathbb{R}^{n}$ such that the angle $\angle P_{1} O P_{2}$ between any two of them and the origin is no smaller than $\theta$. Then for $0<\theta<\pi / 2$,

$$
\frac{1}{n} \log _{2} A(n, \theta) \leq \frac{1+\sin \theta}{2 \sin \theta} \log _{2} \frac{1+\sin \theta}{2 \sin \theta}-\frac{1-\sin \theta}{2 \sin \theta} \log _{2} \frac{1-\sin \theta}{2 \sin \theta}+o(1),
$$

where the convergence of $o(1) \rightarrow 0$ as $n \rightarrow \infty$ is uniform and explicit for $\theta$ within any closed subinterval of $(0, \pi / 2)$. In particular, for $\theta=\pi / 3$, we have

$$
\frac{1}{n} \log _{2} A(n, \theta) \leq 0.40141 \ldots .
$$

Proof. See [KL; cf. also the expositions in [Le] and [CS, Chapter 9].

Remark. For fixed $\theta>\pi / 2$, the function $A(n, \theta)$ is bounded above independently of $n$ : given $k$ unit vectors $v_{1}, v_{2}, \ldots, v_{k}$ separated by angles of $\theta$ or more,

$$
\begin{aligned}
0 & \leq\left\langle v_{1}+\cdots+v_{k}, v_{1}+\cdots+v_{k}\right\rangle \leq k+k(k-1) \max _{i \neq j}\left\langle v_{i}, v_{j}\right\rangle \\
& \leq k+k(k-1) \cos (\theta) .
\end{aligned}
$$

It may hence not be surprising that the derivative of the right side of (3.26) is zero for $\theta=\pi / 2$. This qualitative feature is, in fact, the crucial ingredient in our bound on 3-torsion. In our application, we will apply (3.26) with a $\theta$ that we will have some freedom in choosing. As $\theta$ decreases, the increase in the right-hand side of (3.26) will be offset by a decrease in "cost" linear in $\theta$. In the neighborhood of $\pi / 2$, therefore, it will always be advantageous to decrease $\theta$ slightly.

We shall put this idea into practice in the following way. We shall partition the set of integral points on an elliptic curve so that any two points $P, Q$ in the same part are 
separated by an angle of at least $\theta$ in the Mordell-Weil lattice. We will then apply (3.26) to bound the number of points in each part. (We can do the same for rational points on curves of higher genus; see Section [5) The bounds that correspond to $\theta=\pi / 2$ will correspond (at least in cases where one can bound the difference between canonical and naive heights) to the "uniform" bounds of Bombieri and Pila and of Heath-Brown. Reducing $\theta$ slightly, under favorable circumstances, gives an improvement.

The agreement between the output of this method and the results of $[\mathrm{BP}]$ and HBR is no coincidence: see the remarks after Theorem 3.8 .

3.3. Bounding integral points. In the theorem that follows, the reader might wish to ignore the dependence on $S$ in a first reading. The theorem asserts, in approximate language, that the number of points in $E(K, S)$ of height up to $h_{0}$ is bounded above by $e^{t[K: \mathbb{Q}] h_{0}+(\beta(t)+\epsilon) r}$, where $r$ is the Mordell-Weil rank. Here $t \in[0,1]$ is a free parameter that will be optimized in applications: the basic idea is that if $r$ is small compared to $h_{0}$, it is advantageous to take $t$ small, whereas in applications where $r$ might be very large, we take $t$ close to 1 . This optimization process is formalized in Corollary 3.9.

Roughly speaking, the proof of the theorem proceeds, in words, as follows. We partition the points of $E(K, S)$ into points $\bmod \mathscr{I}$, where $\mathscr{I}$ is a suitable ideal in $\mathscr{O}_{K}$ with norm about $e^{t[K: \mathbb{Q}] h_{0}}$. Proposition 3.4 shows that - after some slight refinement of this partition - the points belonging to the same part are very wellseparated in the Mordell-Weil lattice. We then apply sphere-packing bounds in the form of Proposition 3.7 to each part separately. The term $e^{t[K: \mathbb{Q}] h_{0}}$ arises from the number of parts, whereas the term $e^{\beta(t) r}$ arises from the sphere packing bounds applied to each part. We finally note that the purpose of most of the auxiliary lemmas on previous pages is to help us carry out the "slight refinement" mentioned above.

Theorem 3.8. Let $E$ be an elliptic curve over a number field $K$ defined by a Weierstrass equation (2.4). Let $S$ be a finite set of places of $K$, including all infinite places and all primes dividing the discriminant of $E$.

Then, for every $h_{0} \geq 1$ and every choice of $t \in[0,1]$, the number of $S$-integer points of $E(K)$ of canonical height up to $h_{0}$ is at most

$$
O_{\epsilon,[K: \mathbb{Q}]}\left(C^{s} \epsilon^{-2(s+[K: \mathbb{Q}])} s^{[K: \mathbb{Q}]}\left(1+\log h_{0}\right)^{2} e^{t[K: \mathbb{Q}] h_{0}+(\beta(t)+\epsilon) r}\right),
$$

for every sufficiently small $\epsilon$, where $r$ is the rank of $E(K)$ as a $\mathbb{Z}$-lattice, $s$ is \#S, $C$ is an absolute constant,

$$
\begin{aligned}
\beta(t) & =\frac{1+f(t)}{2 f(t)} \log \frac{1+f(t)}{2 f(t)}-\frac{1-f(t)}{2 f(t)} \log \frac{1-f(t)}{2 f(t)}, \\
f(t) & =\frac{\sqrt{(1+t)(3-t)}}{2} .
\end{aligned}
$$

for $t \in[0,1)$. We set $\beta(1)=0$.

Proof. We first carry out a very mild partitioning (i.e., into very few parts) of $E(K, S)$ so that any two points in the same part have comparable canonical height. Applying Proposition 3.6, we see that one can cover the set $\{P \in E(K, S): \hat{h}(P) \leq$ $\left.h_{0}\right\}$ by $\ll \epsilon^{-1}\left(\log \left(h_{0}\right)+s\right)$ sets of the form $\left\{P \in E(K, S): h_{i} \geq \hat{h}(P) \geq(1-\epsilon) h_{i}\right\}$. 
It therefore suffices to prove the bound (3.28), with $\left(1+\log h_{0}\right)^{2}$ replaced by $(1+$ $\left.\log h_{0}\right)$, just for the set of points $P$ satisfying $(1-\epsilon) h_{0} \leq \hat{h}(P) \leq h_{0}$.

Suppose first that $t \neq 0$. Let $\bar{S}$ be the set of places of $\mathbb{Q}$ below $S$. If $X=$ $\max \left(\left\lceil e^{t h_{0}}\right],(\# \bar{S})^{1+1 /[K: \mathbb{Q}]}, C_{[K: \mathbb{Q}]}\right)$, where $C_{[K: \mathbb{Q}]}$ is an appropiately chosen constant, there is a prime $p$ of $\mathbb{Q}$ with $X \leq p \leq 2 X$ and $p \notin \bar{S}$. The ideal $\mathscr{I}$ of $\mathscr{O}_{K}$ generated by $p$ satisfies

$$
\frac{\log N \mathscr{I}}{[K: \mathbb{Q}]} \geq t h_{0}, \quad N \mathscr{I} \ll_{[K: \mathbb{Q}]} s^{[K: \mathbb{Q}]+1} e^{t[K: \mathbb{Q}] h_{0}} .
$$

The $S$-integer points of $E(K)$ fall into at most $O_{[K: \mathbb{Q}]}(N \mathscr{I})$ classes under reduction modulo $\mathscr{I}$.

Let $R$ be the set of all infinite places and all places of potentially multiplicative reduction. For every $v \in R$, partition $E\left(K_{v}\right)$ into $n_{v}+1$ subsets, where $n_{v}$ is as in (3.1) for $v$ finite and $n_{v}$ is as in (3.5) for $v$ infinite, in both cases with $\epsilon / 2$ instead of $\epsilon$. Consider any tuples $\left(a_{v}\right)_{v \in R},\left(b_{v}\right)_{v \in R}$ with $0 \leq a_{v} \leq n_{v}, b_{v} \in\{0,1\}$. Define $\mathscr{B}$ to be the set of non-torsion points $P \in E(K, S)$ such that, for all $v \in R$, (a) $P \in W_{v, a_{v}}$, (b) $\lambda_{v}(P) \geq 0$ if and only if $b_{v}=1$. We will show how to bound the cardinality of $\mathscr{B}_{h_{0}}=\left\{P \in \mathscr{B}:(1-\epsilon) h_{0} \leq \hat{h}(P) \leq h_{0}\right\}$. Combining this with the fact that the number of sets $\mathscr{B}$ is at most

$$
c_{0}^{s}|\log \epsilon|^{s+[K: \mathbb{Q}]} \epsilon^{-2[K: \mathbb{Q}]}
$$

will yield the conclusion.

Let $M=(S-R) \cup\left\{v \in R: b_{v}=1\right\}$. Let $l: \mathscr{B} \rightarrow \mathbb{R}_{\geq 0}^{M}$ be the map defined by

$$
P \mapsto\left(d_{v} \lambda_{v}(P)\right)_{v \in M} .
$$

Since $\lambda_{v}(P)<0$ for $v \in S-M$, Proposition 3.6 implies

$$
|l(P)|_{1}>[K: \mathbb{Q}] \kappa^{s} \max (1, h(j)) .
$$

On the other hand, by [GS, Proposition 3, (1)], we have that

$$
\sum_{v \notin M} d_{v} \lambda_{v}(P) \geq-\frac{1}{24} h_{K}(j)-3[K: \mathbb{Q}],
$$

and thus $|l(P)|_{1} \leq[K: \mathbb{Q}]\left(h_{0}+3+h(j) / 24\right)$ whenever $P \in \mathscr{B}_{h_{0}}$. By Lemma 3.5. we can cover $l\left(\mathscr{B}_{h_{0}}\right)$ by at most

$$
O\left(c_{1}^{s} \epsilon^{-(s+1)} \log \left(h_{0}+1\right)\right)
$$

balls $B\left(\mathbf{x}, \frac{\epsilon}{8}|\mathbf{x}|_{1}\right)$ in the metric $|\cdot|_{1}$. For $P_{1}, P_{2} \in \mathscr{B}_{h_{0}}$ with $l\left(P_{1}\right), l\left(P_{2}\right) \in B\left(\mathbf{x}, \frac{\epsilon}{8}|\mathbf{x}|_{1}\right)$, we have $\left|l\left(P_{1}\right)-l\left(P_{2}\right)\right|_{1} \leq \frac{\epsilon}{4}|\mathbf{x}|_{1} \leq \frac{\epsilon}{2} \max _{j=1,2}\left|l\left(P_{j}\right)\right|_{1}$. Suppose $P_{1}$ and $P_{2}$ have the same reduction modulo $\mathscr{I}$. Then, by Proposition 3.4.

$$
\hat{h}\left(P_{1}-P_{2}\right) \geq(1-\epsilon) \max _{j=1,2} \hat{h}\left(P_{j}\right)+\frac{\log (N \mathscr{I})}{[K: \mathbb{Q}]} \geq(1+t-\epsilon) \max _{j=1,2} \hat{h}\left(P_{j}\right) .
$$

Embed the Mordell-Weil lattice $E(K)$ modulo torsion into $\mathbb{R}^{\operatorname{rank}(E)}$ so as to send $\hat{h}$ to the square of the Euclidean height. Since $\hat{h}\left(P_{1}\right), \hat{h}\left(P_{2}\right), \hat{h}\left(P_{1}-P_{2}\right)>0$, the images $Q_{1}, Q_{2} \in \mathbb{R}^{\operatorname{rank}(E)}$ of $P_{1}$ and $P_{2}$ are different from each other and from the origin $O$. By (3.33) and the fact that $\hat{h}\left(P_{1}\right), \hat{h}\left(P_{2}\right)$ lie in the interval $\left[(1-\epsilon) h_{0}, h_{0}\right]$, the angle $\angle Q_{1} O Q_{2}$ is at least $\arccos \frac{1-t+O(\epsilon)}{2}$. We may now apply the KL bound (Proposition 3.7), obtaining that there are at most $e^{(\beta(t)+O(\epsilon)) r} \cdot O_{[K: \mathbb{Q}]}(1)$ points of $\mathscr{B}_{h_{0}}$ with image in a given ball $B\left(\mathbf{x}, \frac{\epsilon}{8}|\mathbf{x}|_{1}\right)$ and with prescribed reduction modulo 
$\mathscr{I}$. (The factor $O_{[K: \mathbb{Q}]}(1)$ is an upper bound ( $\mathrm{Me}$ ) on the number of torsion points in $E(K)$.) Combining this with our estimates for the number of possibilities for reduction mod $\mathscr{I}$ (3.30), the number of sets $\mathscr{B}$ (3.31), and the number of balls $B(\mathbf{x}, \ldots)$ (3.32), we obtain the statement of the theorem.

In the case of $t=0$, we proceed as above but without using $\mathscr{I}$.

Remark. Note that $t=0$ gives a pure application of sphere-packing, whereas $t=$ 1 recovers a bound of the quality of $c^{h_{0}}$ (for some constant $c$ ) with almost no dependence on the rank. For our bound on 3-torsion (Theorem 4.2) we will apply the result with $t \in(0,1)$ optimized; for the result on elliptic curves (Theorem 4.5) we will apply it with $t=0$.

The bound with $t=1$ is very closely related to the Bombieri-Pila bound [BP]. To see this, take for a moment $K=\mathbb{Q}$ and let $E$ be given by a Weierstrass equation (2.4). The canonical height of the integral point $P=(x, y)$ on $E$ is given by $\hat{h}(P)=\frac{\log (x)}{2}+O_{E}(1)$; we shall ignore the term $O_{E}(1)$ for the sake of exposition. If $N$ is large, then any integral point $P=(x, y)$ on $E$ with $|x| \leq N,|y| \leq N$ has in fact $|x| \ll N^{2 / 3}$ and thus $\hat{h}(P) \ll \log (N) / 3$. Then the bound given by Theorem 3.8 shows that the number of such points is at most $O\left(N^{1 / 3+\epsilon}\right)$, which agrees with the bound of $[\mathrm{BP}]$ in the case of degree 3 .

This apparent coincidence is a sign of a deeper parallelism between the two methods. Suppose one attempts to carry through the proof of Theorem 3.8 with $t>1$. In other words, we choose the auxiliary ideal $\mathscr{I}$ to satisfy $\log (N \mathscr{I})=$ $1.000001[K: \mathbb{Q}] h_{0}$. In this case, the remark after Proposition 3.7] shows that the number of integral points on $E$ with height $\leq h_{0}$ and reducing to a fixed point modulo $\mathscr{I}$ is bounded independently of the rank of $E(K)$. This is precisely what $\mathrm{BP}$ and $\mathrm{HBR}$ prove, as follows: first, let $L$ be a large integer. One constructs a certain meromorphic function $f$ on $E^{L}$ such that $f$ vanishes to high order along the diagonally embedded $E$. If $P_{1}, \ldots, P_{L}$ all reduce to the same point (modulo $\mathscr{I}$ ), then $\left(P_{1}, \ldots, P_{L}\right) \in E^{L}$ is $\mathscr{I}$-adically near the diagonal, so $f\left(P_{1}, \ldots, P_{L}\right)$ must be divisible by a high power of $\mathscr{I}$. On the other hand, its archimedean norm is not too large; if $L$ and $\mathscr{I}$ are chosen correctly, one obtains thus a contradiction.

Remarkably, the same function $f$ also lurks among our methods. If one were to carry out the proof of Theorem 3.8 with $t>1$ as suggested, using (3.27) instead of sphere-packing, the crucial ingredient is the fact that $\left\langle P_{1}+\cdots+P_{L}, P_{1}+\cdots+\right.$ $\left.P_{L}\right\rangle \geq 0$ for any points $P_{1}, \ldots, P_{L} \in E(K)$. This may be equivalently phrased: $L \sum_{i}\left\langle P_{i}, P_{i}\right\rangle-\sum_{\{i, j\}}\left\langle P_{i}-P_{j}, P_{i}-P_{j}\right\rangle \geq 0$, where the latter sum is taken over unordered subsets $\{i, j\}$ of size 2 .

Now the expression $\left(P_{1}, \ldots, P_{L}\right) \mapsto L \sum_{i=1}^{L}\left\langle P_{i}, P_{i}\right\rangle-\sum_{\{i, j\}}\left\langle P_{i}-P_{j}, P_{i}-P_{j}\right\rangle$ is a Weil height on $E^{L}$ with respect to a certain divisor $D$. (Denoting by $\pi_{i}$ : $E^{L} \rightarrow E, \pi_{i j}: E^{L} \rightarrow E^{2}$ the projections onto the $i$ th and $i j$ th factors, for $i \neq j$, and by $(O)$ and $\Delta$ the divisors on $E$ and $E^{2}$ defined by the origin and diagonal, respectively, we can take $D=L \sum_{i} \pi_{i}^{*}((O))-\sum_{\{i, j\}} \pi_{i j}^{*} \Delta$.) From this point of view, the assertion that this height is always positive is (more or less) the assertion that $D$ is effective, i.e., that there is a meromorphic function $f$ on $E^{L}$ such that $D+(f) \geq 0$. It can be verified that, with appropriate choices, this function can be taken to be the function $f$ discussed above.

One can push this further to an almost word-for-word translation from one method to another. On the other hand, when $t<1$, the proof of Theorem 3.8 
begins to use, in an essential way, the geometry of elliptic curves - one may say: the geometry of curves of non-zero genus - and the translation fails. This is hardly surprising, as the Bombieri-Pila bounds are often tight for rational curves.

Definition 1. We define

$$
\alpha(x)=\min (x t+\beta(t): 0 \leq t \leq 1)
$$

for $x \geq 0$, where $\beta$ is as in (3.29). We set $\alpha(\infty)=\beta(0)$.

Corollary 3.9. Let $E$ be an elliptic curve over a number field $K$. Let $S$ be a finite set of places of $K$, including all infinite places and all primes dividing the discriminant of $E$. Let $R \geq \max \left(1, \operatorname{rank}_{\mathbb{Z}} E(\mathbb{Q})\right)$. Then, for every $h_{0} \geq 1$, the number of $S$-integer points of $E(K)$ of canonical height up to $h_{0}$ is at most

$$
O_{\epsilon,[K: \mathbb{Q}]}\left(C^{s} \epsilon^{-2(s+[K: \mathbb{Q}])} s^{[K: \mathbb{Q}]}\left(1+\log h_{0}\right)^{2} e^{R \cdot \alpha\left(\frac{h_{0}[K: \mathbb{Q}]}{R}\right)+\epsilon R}\right)
$$

for every sufficiently small $\epsilon$, where $s$ is $\# S$ and $C$ is an absolute constant.

Proof. The statement is simply that of Theorem 3.8 with $t$ optimized.

Remark. Let $S$ to be the set of all infinite places and all primes of bad reduction, and assume, for simplicity, that $K=\mathbb{Q}$. Assume that $h_{0}>c \max (\log \Delta, h(j))$ for some constant $c$. Then the main contribution to 3.35 is given by

$$
e^{R \cdot \alpha\left(h_{0} / R\right)}
$$

Since $\beta^{\prime}(1)=0$, the minimum of $x t+\beta(t)$ is attained to the left of $t=1$. Since $h_{0}>c \log \Delta \gg R$, we actually have $\alpha\left(h_{0} / R\right)<\left(1-\delta_{0}\right) h_{0} / R$ for some constant $\delta_{0}>0$ depending only on $c$. We obtain a bound of the type

$$
\# E(K, S) \ll e^{\left(1-\delta_{1}\right) h_{0}}
$$

for any $\delta_{1}<\delta_{0}$. As remarked after Theorem 3.8, the bound $e^{h_{0}}$ would be obtained if we proceeded as in $\mathrm{BP}$ and $[\mathrm{HBR}$; thus $[3.36$ gives an improvement in the exponent.

3.4. Quantitative consequences of bounds on the height. There is a long tradition - starting with $[\mathrm{Ba}$ - of effective upper bounds on the height of integral points on an elliptic curve. It is clear that any such bound yields a quantitative result, i.e., an effective upper bound on the number of integral points.

We will see how upper bounds on heights can be combined with pure quasiorthogonality so as to show that $\# E(K, S)$ is essentially bounded by a power of the discriminant $\Delta$ of $E$. There are already bounds of a comparable quality in the literature; in particular, ES can be used to bound $\# E(\mathbb{Q},\{\infty\})$ by a power of $\Delta$. What we have here is simply an improvement in the exponent. In the next section, we will be in a situation in which exponents are crucial; we will also be able to take advantage of complex multiplication to reduce our exponents further.

We note that for our purposes it is very important that the available bounds for integral points have the property that they bound the canonical height by a power (or at least a sub-exponential function) of the coefficients of the elliptic curve. In our context we will use a very strong bound due to Hajdu and Herendi $\mathrm{HjHr}$.

In what follows we take $K=\mathbb{Q}$ for simplicity. 
Proposition 3.10. Let $E$ be an elliptic curve over $\mathbb{Q}$ defined by a Weierstrass equation of the form $y^{2}=x^{3}+a x+b$, where $a, b \in \mathbb{Z}$. Let $S$ be a finite set of places of $\mathbb{Q}$, including the infinite place. Then, for any $P \in E(\mathbb{Q}, S)$,

$$
\hat{h}(P) \leq c_{1} p^{c_{2}}(1+\log p)^{c_{3}(s+1)}(s+1)^{c_{4}(s+1)}|\Delta|^{c_{5}} \log H_{E}
$$

where $s=\# S, p$ is the largest prime in $S$ (set $p=1$ if $S=\infty$ ), $\Delta$ is the discriminant of $E, H_{E}=\max (|a|,|b|)$, and $c_{1}, c_{2}, \ldots, c_{5}$ are explicit absolute constants.

Proof. See $\mathrm{HjHr}$, Theorem 2]. See [Sil3, for bounds on $\left|\hat{h}(P)-\frac{1}{2} h(x(P))\right|$.

Corollary 3.11. Let $E$ be an elliptic curve over $\mathbb{Q}$ defined by a Weierstrass equation with integer coefficients. Let $S$ be a finite set of places of $\mathbb{Q}$, including $\infty$ and all primes dividing the discriminant of $E$. Then the number of $S$-integer points on $E(\mathbb{Q})$ is at most

$$
O_{\epsilon}\left(C^{s} \epsilon^{-2(s+1)}(\log |\Delta|+\log p)^{2} e^{(\beta(0)+\epsilon) r}\right)
$$

for every sufficiently small $\epsilon$, where $r$ is the rank of $E(\mathbb{Q})$ as a $\mathbb{Z}$-lattice, $s$ is $\# S$, $C$ is an absolute constant, $p$ is the largest prime in $S, \Delta$ is the discriminant of $E$, and $\beta(t)$ is as in (3.29). Numerically, $\beta(0)=0.2782 \ldots$.

Proof. We may, without loss of generality, assume that $E$ is given by $y^{2}=x^{3}+a x+b$ with $a, b \in \mathbb{Z}$. (Indeed, we may make a linear substitution of variables transforming $E$ to this form, carrying integer points to integer points and increasing $\log |\Delta|$ by at most an absolutely bounded amount.) The result is then immediate from Theorem 3.8 and Proposition 3.10, (Note that $\log \log H_{E} \ll \log |\Delta|$, by Proposition 3.10 itself applied to $y^{2}=x^{3}-27 \Delta$.)

Corollary 3.12. Let $E$ be an elliptic curve over $\mathbb{Q}$ defined by a Weierstrass equation with integer coefficients. Then the number of integer points on $E(\mathbb{Q})$ is at most

$$
O_{\epsilon}\left(|\Delta|^{\frac{\beta(0)}{2 \log 2}+\epsilon}\right)
$$

for every sufficiently small $\epsilon$, where $\Delta$ is the discriminant of $E$ and $\beta(t)$ is as in (3.29). Numerically, $\frac{\beta(0)}{2 \log 2}=0.20070 \ldots$.

Proof. We can take $E$ to be given by an equation of the form $y^{2}=x^{3}+a x+b$, $a, b \in \mathbb{Z}$. Let $\epsilon_{0}$ be sufficiently small. By Corollary 3.11, we obtain a bound of

$$
O_{\epsilon_{0}}\left(|\Delta|{ }^{\epsilon_{0}} \epsilon_{0}^{-2(\omega(\Delta)+1)} \log (|\Delta|)^{2} e^{\left(\beta(0)+\epsilon_{0}\right) r}\right),
$$

where $r$ is the rank of $E(K)$. Let $K$ be the cubic field generated by a root of $x^{3}+a x+b=0$. Then $r \leq \log _{2} h_{2}(K)+o(\log |\Delta|)$ by BK, Proposition 7.1]. (If $x^{3}+a x+b$ is not irreducible, a stronger bound follows by [Ma, Proposition 9.8(b)].) Since the discriminant of $K$ divides $\Delta$, we see that $h_{2}(K) \leq h(K) \ll \Delta^{1 / 2+\epsilon_{0}}$. Finally, $2(\omega(\Delta)+1)<\epsilon_{0} \log |\Delta|$ for $|\Delta|$ large enough, and thus $\epsilon_{0}^{-2(\omega(\Delta)+1)}<$ $|\Delta|^{\log \epsilon_{0} \mid \epsilon_{0}}$. We set $\epsilon_{0}$ small enough in terms of $\epsilon$, and we are done.

Remark. Corollary 3.12 improves on the bound $O_{\epsilon}\left(|\Delta|^{1 / 2+\epsilon}\right)$ proven by W. Schmidt ([Schm, Theorem 1]) on the basis of the results in [ES]. The exponent $1 / 2$ arises from the trivial bound $h_{2}(L) \leq h(L) \ll_{\epsilon} \Delta^{1 / 2+\epsilon}$, where $L$ is a cubic field over $\mathbb{Q}$ of discriminant $\Delta$. 
One of our main tasks in the following section will be to do better than Corollary 3.12 in the case of Mordell equations. We have not been able to improve on $h_{2}(L) \ll$ $\Delta^{1 / 2+\epsilon}$, but, as we will see, we can improve on $h_{3}(\mathbb{Q}(\sqrt{D})) \ll D^{1 / 2+\epsilon}$. Note that Corollary 3.12 would already be enough to break current bounds on the number of elliptic curves of given conductor (cf. Theorem 4.5)).

\section{Elliptic CURVES AND 3-TORSiON}

Throughout this section, let $D$ be a nonzero integer. We denote by $E_{D}$ the elliptic curve $y^{2}=x^{3}+D$. Suppose, for simplicity, that $D$ is negative; as we will see, we can assume as much by Scholz's reflection principle. We may bound the class number $h_{3}\left(\mathbb{Q}(\sqrt{D})\right.$ ) from above by the number of integer points on $E_{D \delta^{2}}$, $1 \leq \delta \ll|D|^{1 / 4}$. We then apply Corollary 3.9 to bound the number of integer points in terms of the rank of $E_{D \delta^{2}}$. Since $E_{D \delta^{2}}$ has complex multiplication, one may do a CM-descent and thereby bound the rank of $E_{D \delta^{2}}$ in terms of $h_{3}(\mathbb{Q}(\sqrt{D}))$.

We thus establish a feedback that, once started, lowers $h_{3}(\mathbb{Q}(\sqrt{D}))$ to an equilibrium point. Note that Theorem 3.8 with $t=0$ (or $t=1$ ) would be insufficient to start the loop; only a mixed bound will do where a pure bound will not.

The problem of counting elliptic curves of given conductor also reduces to counting points on curves of the form $E_{D \delta^{2}}$. Again, their rank may be bounded by means of a CM-descent, and the new bounds on $h_{3}(\mathbb{Q}(\sqrt{D}))$ can thus be applied.

\subsection{Bounds for 3-torsion and cubic fields of fixed discriminant.}

Lemma 4.1. The rank of $E_{D}(\mathbb{Q})$ satisfies

$$
\operatorname{rank}_{\mathbb{Z}} E_{D}(\mathbb{Q}) \leq A+B \omega(D)+2 \log _{3} h_{3}(\mathbb{Q}(\sqrt{D}))
$$

for some absolute constants $A, B$.

Proof. See, e.g., [Fo, Proposition 2].

Theorem 4.2. Let $D$ be a positive integer. Suppose $-D$ is a fundamental discriminant. Then, for every $\epsilon>0$,

$$
h_{3}(\mathbb{Q}(\sqrt{-D})) \ll_{\epsilon} D^{\lambda+\epsilon}, \quad h_{3}(\mathbb{Q}(\sqrt{3 D})) \ll_{\epsilon} D^{\lambda+\epsilon},
$$

where $\lambda$ is the unique real solution in the range $\lambda \in(0.4,0.5)$ to the equation

$$
\lambda=1 / 4+\frac{2 \lambda}{\log (3)} \alpha\left(\frac{\log (3)}{8 \lambda}\right) .
$$

Numerically, $\lambda=0.44178 \ldots$

Proof. Let $\iota$ be an embedding of $\mathbb{Q}(\sqrt{-D})$ into $\mathbb{C}$. Let $\mathfrak{a}$ be an ideal of $\mathscr{O}_{\mathbb{Q}(\sqrt{-D})}$. Then $\iota(\mathfrak{a})$ is a lattice in $\mathbb{C}$ of covolume $N(\mathfrak{a}) D^{1 / 2}$. Minkowski's theorem (see [Si]) shows that $\iota(\mathfrak{a})$ contains $x \in \mathbb{C}$ with $|x| \ll N(\mathfrak{a})^{1 / 2} D^{1 / 4}$, the implicit constant being absolute. This implies that $\mathfrak{a}$ contains an element $\alpha$ of norm $\ll N(\mathfrak{a}) \sqrt{D}$. Then $\mathfrak{a}^{-1} \cdot \alpha$ is an integral ideal in the same ideal class as $\mathfrak{a}^{-1}$ but of norm $\ll D^{1 / 2}$.

In particular, any 3 -torsion class in the ideal class group of $\mathbb{Q}(\sqrt{-D})$ has a representative $\mathfrak{a}$ that satisfies $N(\mathfrak{a}) \ll D^{1 / 2}$. Since $\mathfrak{a}^{3}$ is principal, it follows that $\mathfrak{a}^{3}=(y+\delta \sqrt{-D})$ where $y+\delta \sqrt{-D} \in \mathscr{O}_{\mathbb{Q}(\sqrt{-D})}$. Thus $N(\mathfrak{a})^{3}=y^{2}+D \delta^{2}$. Since $y+\delta \sqrt{-D}$ is an integer in $\mathbb{Q}(\sqrt{-D})$, we know that $2 y$ and $2 \delta$ are integers. 
Since $N(\mathfrak{a}) \ll D^{1 / 2}$, the 3 -torsion class represented by $\mathfrak{a}$ has given us a solution to

(4.3) $(4 x)^{3}=(8 y)^{2}+D(8 \delta)^{2}\left(4 x, 8 y, 8 \delta \in \mathbb{Z},|x| \ll D^{1 / 2},|y| \ll D^{3 / 4},|\delta| \ll D^{1 / 4}\right)$.

Conversely, any solution to (4.3) determines $\mathfrak{a}$ up to $\ll D^{\epsilon}$ possibilities. We have therefore deduced that $h_{3}(\mathbb{Q}(\sqrt{-D}))$ is at most a constant times

$$
D^{1 / 4+\epsilon} \max _{|\delta| \ll D^{1 / 4}} \#\left\{(x, y) \in E_{-D \delta^{2}}(\mathbb{Q},\{\infty\}):|x| \ll D^{1 / 2},|y| \ll D^{3 / 4}\right\} .
$$

The curve $E_{-D \delta^{2}}$ is a twist of $E_{-1}$, and the map $(x, y) \rightarrow\left(\frac{x}{D^{1 / 3} \delta^{2 / 3}}, \frac{y}{D^{1 / 2} \delta}\right)$ gives an isomorphism $E_{-D \delta^{2}} \rightarrow E_{-1}$ over $\overline{\mathbb{Q}}$. Thus the difference $\left|\hat{h}(P)-\frac{1}{2} h\left(\frac{x(P)}{D^{1 / 3} \delta^{2 / 3}}\right)\right|$ is bounded above by a constant for all $P \in E(\overline{\mathbb{Q}})$; on the other hand

$$
\frac{1}{2} h\left(x(P) D^{-1 / 3} \delta^{-2 / 3}\right)=\frac{1}{6} h\left(\frac{x(P)^{3}}{D \delta^{2}}\right) \leq \max \left(\frac{1}{2} \log |x(P)|, \frac{1}{6} \log \left|D \delta^{2}\right|\right) .
$$

Thus any point $P=(x, y) \in E_{-D \delta^{2}}$ satisfying (4.4) has $\hat{h}(P) \leq \frac{\log (D)}{4}+O(1)$.

Let $\gamma=\limsup _{D \rightarrow \infty} \frac{\log \left(h_{3}(\mathbb{Q}(\sqrt{-D}))\right)}{\log (D)}$. Lemma 4.1 shows that, for $D$ large enough and any $\delta \ll D^{1 / 4}$,

$$
\operatorname{rank}_{\mathbb{Z}} E_{-D \delta^{2}}(\mathbb{Q}) \leq R=\log (D)\left(\frac{2 \gamma}{\log (3)}+o(1)\right) .
$$

We apply Corollary 3.9 with $S=\{\infty\} \cup\left\{p: p \mid 6 D \delta^{2}\right\}$ and $h_{0}=\log (D) / 4+O(1)$, obtaining

$$
\#\left\{P \in E_{-D \delta^{2}}(\mathbb{Q}, S): \hat{h}(P) \leq h_{0}\right\} \ll_{\epsilon} D^{\frac{2 \gamma}{\log 3} \alpha\left(\frac{\log (3)}{8 \gamma}\right)+\epsilon}
$$

for every $\epsilon>0$.

By (4.4) and (4.6), we conclude that

$$
\gamma \leq \frac{1}{4}+\frac{2 \gamma}{\log 3} \alpha\left(\frac{\log 3}{8 \gamma}\right) .
$$

One has the a priori bound $\gamma \leq 1 / 2$. We iterate (4.7). Apply Scholz's reflection principle $(\underline{\mathrm{Sch}})$ to obtain $h_{3}(\mathbb{Q}(\sqrt{3 D})) \ll D^{\lambda+\epsilon}$ therefrom.

Corollary 4.3. The number of cubic extensions of $\mathbb{Q}$ of discriminant $D$ is $O\left(|D|^{\lambda+\epsilon}\right)$, where $\lambda$ is as in Theorem 4.2 .

Proof. This is an immediate consequence of Theorem 4.2, see [Ha, Satz 7].

Remark. The best previously known bound was the trivial one, namely that $h_{3}(\mathbb{Q}(\sqrt{-D})) \leq h(\mathbb{Q}(\sqrt{-D})) \ll_{\epsilon} D^{1 / 2+\epsilon}$. The conditional results known to the authors are as follows. S. Wong has shown (Wo2 ) that the Birch-Swinnerton-Dyer conjecture, together with the Riemann hypothesis for the $L$-functions of elliptic curves, implies that $h_{3}(\mathbb{Q}(\sqrt{-D})) \ll_{\epsilon} D^{1 / 4+\epsilon}$.

Let $\chi_{D}$ be the quadratic Dirichlet character associated to $\mathbb{Q}(\sqrt{-D})$. Then the Riemann hypothesis for $L\left(s, \chi_{D}\right)$ alone implies $h_{3}(\mathbb{Q}(\sqrt{-D})) \ll_{\epsilon} D^{1 / 3+\epsilon}$. We sketch the proof communicated to us by Soundararajan; see also the remark at the end of So.

Assume $D \equiv 1 \bmod 4$ for simplicity. Let $\sigma$ be the Galois automorphism of $K=\mathbb{Q}(\sqrt{-D})$ over $\mathbb{Q}$. Assuming the Riemann hypothesis for $L\left(s, \chi_{D}\right)$, one sees that there are $\gg_{\epsilon} D^{1 / 6-\epsilon}$ primes $p$ with $p<D^{1 / 6}$ and $\chi_{D}(p)=1$; equivalently, there are $\gg_{\epsilon} D^{1 / 6-\epsilon}$ prime ideals $\mathfrak{p}$ of $\mathscr{O}_{K}$ with $N \mathfrak{p}<D^{1 / 6}$ and $N \mathfrak{p}$ prime. If 
two such distinct ideals $\mathfrak{p}_{1}, \mathfrak{p}_{2}$ represented the same class in the quotient group $\mathrm{Cl}\left(\mathscr{O}_{K}\right) / \mathrm{Cl}\left(\mathscr{O}_{K}\right)[3]$, then $\mathfrak{p}_{1}^{\sigma} \mathfrak{p}_{2}$ would represent a 3 -torsion ideal class; in particular, $N\left(\mathfrak{p}_{1}^{\sigma} \mathfrak{p}_{2}\right)^{3}$ would be, as in the proof of Theorem 4.2. an integer of the form $x^{2}+D y^{2}$. Since $N\left(\mathfrak{p}_{1}^{\sigma} \mathfrak{p}_{2}\right)^{3}<D$, this forces $y=0$, leading to a contradiction. This shows that $\#\left(\mathrm{Cl}\left(\mathscr{O}_{K}\right) / \mathrm{Cl}\left(\mathscr{O}_{K}\right)[3]\right) \gg_{\epsilon} D^{1 / 6-\epsilon}$, which gives $\#\left(\mathrm{Cl}\left(\mathscr{O}_{K}\right)[3]\right) \ll_{\epsilon} D^{1 / 3+\epsilon}$ as desired.

4.2. Elliptic curves of given conductor. The following is well known; see, e.g., BS, proof of Theorem 1].

Lemma 4.4. Let $S$ be a set of finite places of $\mathbb{Q}$. There is a map from the set of all isomorphism classes of elliptic curves over $\mathbb{Q}$ with good reduction outside $S \cup\{2,3\}$ to the union $\bigcup_{C} E_{C}(\mathbb{Q}, S \cup\{2,3\})$ of the sets of $(S \cup\{2,3\})$-integer points on each of the curves

$$
E_{C}: y^{2}=x^{3}+C,
$$

where $C=\prod_{p \in S \cup\{2,3\}} p^{a_{p}}, 0 \leq a_{p} \leq 5$. Each fiber has size at most $2^{\# S+3}$.

Proof. See [BS, p. 100].

Theorem 4.5. The number of elliptic curves over $\mathbb{Q}$ of conductor $N$ is

$$
O_{\epsilon}\left(N^{\gamma+\epsilon}\right)
$$

for every $\epsilon>0$, where $\gamma=\frac{2 \lambda \cdot \beta(0)}{\log 3}, \beta$ is as in Theorem 3.8 and $\lambda$ is as in (4.2); numerically, $\gamma=0.22377 \ldots$.

Proof. Let $S=\{p: p \mid N\}, M=\prod_{p \in S} p$. In view of Lemma 4.4,

$$
\#\{E / \mathbb{Q}: E \text { has good reduction outside of } S\} \ll 6^{2 \# S} \max _{C} \# E_{C}(\mathbb{Q}, S),
$$

where the maximum is taken over all $C=\prod_{p \in S \cup\{2,3\}} p^{a_{p}}, 0 \leq a_{p} \leq 5$.

By Corollary 3.11. Lemma 4.1, and Theorem 4.2 we obtain

$$
\begin{aligned}
\max _{C} \# E_{C}(\mathbb{Q}, S) & \ll_{\epsilon} N^{\epsilon} \max _{C} e^{\operatorname{rank}\left(E_{C}(\mathbb{Q})\right) \cdot(\beta(0)+\epsilon)} \\
& \ll_{\epsilon} N^{\epsilon} e^{2 \log _{3}\left(h_{3}(\mathbb{Q}(\sqrt{-N}))\right)(\beta(0)+\epsilon)} \ll_{\epsilon} N^{2 \beta(0) \lambda / \log 3+\epsilon} .
\end{aligned}
$$

Remark. The above argument shows that, on any Mordell curve $E: y^{2}=x^{3}+D$, where $D$ is a rational integer, there are at most $O\left(D^{0.22377 \ldots}\right)$ integer points. Notice the improvement over Corollary 3.12 . We are using, of course, the fact that Mordell curves have complex multiplication.

\section{Rational points: Beyond 2/d}

The technique here is also applicable to counting rational points on curves of genus $\geq 1$. This will be pursued in more detail in a separate paper; here we content ourselves with indicating, in an approximate fashion, how one can use the method of this paper to bound the number of points on a curve of higher genus without knowing the rank of its Jacobian. Recall that Heath-Brown HBR has shown that if $C$ is (for example) a plane irreducible curve of degree $d$, then the number of points in $C(\mathbb{Q})$ of naive height $\leq H_{0}$ is $O_{d, \epsilon}\left(H_{0}^{2 / d+\epsilon}\right)$; Elkies independently proved a related bound [El] with a view to algorithmic applications. 
The method of this paper, roughly speaking, recovers the exponent $2 / d$ for curves of higher genus, provided that we may completely ignore the factors of $O(1)$ that arise when dealing with Weil height functions. When further simplifications are valid, the procedure delivers an exponent lower than $2 / d$.

Let $C$ be a proper smooth curve of genus $\geq 1$ over a number field $K$. To further simplify matters, let us assume that $C$ has a $K$-rational point $a$. The factors of $O(1)$ that occur in the computations below depend both on $C$ and $a$. Let $h_{a}: C(\bar{K}) \rightarrow \mathbb{R}$ be a Weil height with respect to the divisor $(a)$, and suppose we are interested in bounds for the number of points $P \in C(K)$ with $h_{a}(P) \leq h_{0}$. Note that here $h_{a}$ will denote a Weil height "over $K$ ", not an absolute height normalized by a factor $\frac{1}{[K: \mathbb{Q}]}$ (see the difference between (2.2) and (2.3)). For complete conformity with our previous notation we should denote it $h_{a, K}$, but we shall suppress the $K$ subscript for typographical ease.

Let $J$ be the Jacobian of $C$. Let $j_{a}: C \rightarrow J$ be the embedding that sends $P \in C$ to $P-a \in \operatorname{Pic}^{0}(C)$, and let $\Theta$ be the associated theta-divisor, i.e., $j_{a}(C)+$ $j_{a}(C)+\cdots+j_{a}(C)$, taken $g-1$ times. Let $\Delta \in C \times C$ be the diagonal and $h_{\Delta}: C(\bar{K}) \times C(\bar{K}) \rightarrow \mathbb{R}$ an associated Weil height.

We denote by $\langle\cdot, \cdot\rangle_{\Theta}$ the inner product on $J(\bar{K})$ induced by the canonical height associated to $\Theta$, which agrees up to $O(1)$ with the Weil height associated to the symmetric divisor $\frac{1}{2}\left(\Theta+[-1]^{*} \Theta\right) \in \operatorname{Pic}(C) \otimes_{\mathbb{Z}} \mathbb{R}$. (Cf. [HS, B.5]; the map $z \rightarrow\langle z, z\rangle_{\Theta}$ on $J(\bar{K})$ is the map $\hat{q}_{J, \Theta}$ in the notation of [HS, Theorem B.5.6].)

We set $\|z\|_{\Theta}^{2}=\langle z, z\rangle_{\Theta}$ for $z \in J(\bar{K})$. Note that for $x \in C(\bar{K})$

$$
\left\|j_{a}(x)\right\|_{\Theta}^{2}=g h_{a}(x)+O\left(\sqrt{1+h_{a}(x)}\right)
$$

(this follows from [HS, Theorem B.5.9], since $j_{a}^{*} \Theta$ is algebraically equivalent to $g \cdot(a)$; cf. [HS, Theorem A.8.2.1]). By the proof of Mumford's gap principle (see [HS, Theorems A.8.2.1 and B.6.5]), one has, for any $x, y \in C(\bar{K})$,

$$
2\left\langle j_{a}(x), j_{a}(y)\right\rangle_{\Theta}=h_{a}(x)+h_{a}(y)-h_{\Delta}(x, y)+O(1) .
$$

Now suppose that $x, y \in C(K)$ are chosen so that $h_{a}(x), h_{a}(y) \leq h_{0}$. The theory of local heights ( $\mathrm{Se} 2$ ) shows that, if $x \neq y \in C(K)$ reduce to the same point modulo $\mathfrak{p}$, any prime ideal of $\mathscr{O}_{K}$, then $h_{\Delta}(x, y) \geq \log (N \mathfrak{p})+O(1)$. Indeed, the hypothesis guarantees that $(x, y)$ is $\mathfrak{p}$-adically close to the diagonal, which forces $h_{\Delta}(x, y)$ to be large.

For such $x, y$, (5.2) yields $2\left\langle j_{a}(x), j_{a}(y)\right\rangle_{\Theta} \leq 2 h_{0}-\log (N \mathfrak{p})+O(1)$. Thus, if we choose $\mathfrak{p}$ so that $\log (N \mathfrak{p})>2(1+\epsilon) h_{0}$, we have $\left\langle j_{a}(x), j_{a}(y)\right\rangle_{\Theta} \leq-\epsilon h_{0}+O(1)$.

On the other hand, in view of (5.1), we have $\max \left(\left\|j_{a}(x)\right\|_{\Theta}^{2},\left\|j_{a}(y)\right\|_{\Theta}^{2}\right)<g h_{0}+$ $O\left(1+\sqrt{h_{0}}\right)$. The angle $\theta_{x y}$ between the points $j_{a}(x), j_{a}(y)$ in (the Mordell-Weil lattice of) $J(K)$ thus satisfies

$$
\cos \left(\theta_{x y}\right) \leq \frac{-\epsilon h_{0}+O(1)}{g h_{0}+O\left(1+\sqrt{h_{0}}\right)}
$$

Now, for the sake of the exposition, let us ignore the factors $O(1)$ and $O\left(\sqrt{1+h_{0}}\right)$ in (5.3). It then follows that, if $\log (N \mathfrak{p})=2(1+\epsilon) h_{0}$, then $\cos \left(\theta_{x y}\right) \leq-\epsilon / g$.

For reasons outlined in the remark following Proposition [3.7 the number of vectors in $\mathbb{R}^{N}$ all of whose mutual angles satisfy $\cos (\theta) \leq-\epsilon$ is bounded by $O_{\epsilon}(1)$. It follows that the number of points $P \in C(K)$ of height $h_{a}(P) \leq h_{0}$ that reduce to a fixed point in $C\left(\mathscr{O}_{K} / \mathfrak{p}\right)$ is $O_{\epsilon}(1)$; in particular, the number of points $P \in C(K)$ of height $h_{a}(P) \leq h_{0}$ is $\ll_{\epsilon} N \mathfrak{p}=\exp \left(2(1+\epsilon) h_{0}\right)$. 
To recognize the exponent, note that if $C$ is a curve of degree $d$ in a projective space $\mathbb{P}^{n}$, then the naive (exponential) height $H_{\mathbb{P}^{n}}$ on $C$ satisfies $\log H_{\mathbb{P}^{n}}-d h_{a}=$ $O\left(1+\sqrt{h_{a}}\right)$. Thus, the number of points $P \in C(K)$ with $H_{\mathbb{P}^{n}} \leq H_{0}$ is $\ll H_{0}^{2 / d+\epsilon}$, recovering Heath-Brown's result.

Furthermore, one can "perturb" this method by decreasing $N \mathfrak{p}$, as was carried out in the text for integral points on elliptic curves; a small enough perturbation improves the exponent $2 / d$. This has been carried out in $[\mathrm{EV}]$, which incorporates also some different ideas stemming from the work of Heath-Brown [HBR].

\section{ACKNOWLEDGMENTS}

We would like to thank M. Bhargava, A. Brumer, S. David, F. Gerth, D. Goldfeld, R. Heath-Brown, H. Iwaniec, A. J. de Jong, L. B. Pierce, J. H. Silverman and K. Soundararajan for their advice and encouragement.

\section{REFERENCES}

[Ba] Baker, A., The diophantine equation $y^{2}=a x^{3}+b x^{2}+c x+d$, J. Lond. Math. Soc. 43 (1968), 1-9. MR0231783 (38:111)

[BEG] Brindza, B., Evertse, J.-H., and K. Györy, Bounds for the solutions of some Diophantine equations in terms of discriminants, J. Austral. Math. Soc. Ser. A 51 (1991), no. 1, 8-26. MR.1119684 (92e:11024)

[BK] Brumer, A., and K. Kramer, The rank of elliptic curves, Duke Math. J. 44 (1977), 715-743. MR0457453 (56:15658)

[BP] Bombieri, E., and J. Pila, The number of integral points on arcs and ovals, Duke Math. J. 59 (1989), no. 2, 337-357. MR1016893(90j:11099)

[BS] Brumer, A., and J. H. Silverman, The number of elliptic curves over $\mathbb{Q}$ with conductor $N$, Manuscripta Math. 91 (1996), no. 1, 95-102. MR1404420(97e:11062)

[Bu] Bugeaud, Y., Bounds for the solutions of superelliptic equations, Comp. Math. 107 (1997), 187-219. MR1458749 (98c:11025)

[CS] Conway, J. H., and N. J. A. Sloane, Sphere packings, lattices and groups, Grundlehren der Mathematischen Wissenschaften, 290, Springer-Verlag, New York, 1988. MR0920369 (89a:11067)

[Da] David, S., Points de petite hauteur sur les courbes elliptiques, J. Number Theory 64 (1997), no. 1, 104-129. MR1450488 (98k:11067)

[Du] Duke, W., Bounds for arithmetic multiplicities, Proceedings of the International Congress of Mathematicians, Vol. II (Berlin, 1998), Doc. Math. 1998, extra vol. II, 163-172. MR:1648066 (99j:11042)

[DK] Duke, W., and E. Kowalski, A problem of Linnik for elliptic curves and mean-value estimates for automorphic representations; with an appendix by Dinakar Ramakrishnan, Invent. Math. 139 (2000), no. 1, 1-39. MR.1728875 (2001b:11034)

[El] Elkies, N.D., Rational points near curves and small $\left|x^{3}-y^{2}\right|$ via lattice reduction, Algorithmic Number Theory, 33-63, Lecture Notes in Computer Science, 1838, Springer-Verlag, Berlin, 2000. MR,1850598 (2002g:11035)

[EV] Ellenberg, J. and A. Venkatesh, On uniform bounds for rational points on non-rational curves. IMRN 35 (2005).

[ES] Evertse, J.-H., and J. H. Silverman, Uniform bounds for the number of solutions to $y^{n}=f(x)$, Math. Proc. Cambridge Philos. Soc. 100 (1986), no. 2, 237-248. MR0848850 $(87 \mathrm{k}: 11034)$

[Fo] Fouvry, É, Sur le comportement en moyenne du rang des courbes $y^{2}=x^{3}+k$, Séminaire de Théorie des Nombres, Paris, 1990-91, Progr. Math., 61-84, Birkhäuser Boston, Boston, MA, 1993. MR1263524 (95b:11057)

[GS] Gross, R., and J. H. Silverman, S-integer points on elliptic curves, Pacific J. Math. 167 (1995), no. 2, 263-288. MR 1328329 (96c:11057)

[HjHr] Hajdu, L., and T. Herendi, Explicit bounds for the solutions of elliptic equations with rational coefficients, J. Symbolic Comput. 25 (1998), no. 3, 361-366. MR1615334 (99a:11033) 
[Ha] Hasse, H., Arithmetische Theorie der kubischen Zahlkörper auf klassenkörpertheoretischer Grundlage, Math. Z. 31 (1930) 565-582. Corrigendum: Math. Z. 31 (1930) 799. MR1545136

[HBR] Heath-Brown, R., The density of rational points on curves and surfaces, Ann. of Math. (2) 155 (2002), no. 2, 553-595. MR1906595 (2003d:11091)

[He] Helfgott, H. A., On the square-free sieve, Acta Arith. 115 (2004), no. 4, 349-402. MR2099831 (2005h:11211)

[Her] Herrmann, E., Bestimmung aller S-ganzen Lösungen auf elliptischen Kurven, Ph.D. thesis, Universität des Saarlandes.

[HS] Hindry, M., and J. H. Silverman, Diophantine geometry, Springer-Verlag, New York, 2000. MR.1745599(2001e:11058)

[KL] Kabatjanskii, G. A., and V. I. Levenshtein, Bounds for packings on the sphere and in space, Problemy Peredači Informacii 14 (1978), no. 1, 3-25. MR0514023 (58:24018)

[KT] Kotov, S. V., and L. A. Trelina, S-ganze Punkte auf elliptischen Kurven, J. Reine Angew. Math. 306 (1979), 28-41. MR0524645 (80b:10024)

[La] Lang, S., Elliptic Curves: Diophantine Analysis, Springer-Verlag, 1978. MR0518817 (81b:10009)

[Le] Levenshtein, V. I., Universal bounds for codes and designs, Handbook of coding theory, North-Holland, Amsterdam, Vol. I, 499-648. MR.1667942

[Ma] Mazur, B., Rational points of abelian varieties with values in towers of number fields, Invent. Math. 18 (1972), 183-266. MR0444670 (56:3020)

[Me] Merel, L., Bornes pour la torsion des courbes elliptiques sur les corps de nombres, Invent. Math. 124 (1996), no. 1-3, 437-449. MR1369424 (96i:11057)

[Mu] Mumford, D., A remark on Mordell's conjecture, Amer. J. Math. 87 (1965), 1007-1016. MR0186624(32:4083)

[Mur] Murty, M. R., Exponents of class groups of quadratic fields, Topics in number theory, 229-239, Kluwer Academic, Dordrecht, 1997. MR1691322 (2000b:11123)

[Nek] Nekováŕ, J., Class numbers of quadratic fields and Shimura's correspondence, Math. Ann. 287 (1990), no. 4, 577-594. MR1066816 (91k:11051)

[Pi] Pierce, L. B., The 3-part of class numbers of quadratic fields, J. London Math. Soc. (2) 71 (2005), no. 3, 579-598. MR2132372

[Pin] Pintér, A., On the magnitude of integer points on elliptic curves, Bull. Austral. Math. Soc. 52 (1995), no. 2, 195-199. MR.1348477 (96k:11070)

[Schm] Schmidt, W., Integer points on curves of genus 1, Compositio Math. 81 (1992), 33-59. MR 1145607 (93e:11076)

[Sch] Scholz, A., Uber die Beziehung der Klassenzahlen quadratischer Körper zueinander, J. Reine Angew. Math. 166 (1932), 201-203.

[Se] Serre, J.-P., Lectures on the Mordell-Weil theorem, 3rd ed., Vieweg, Braunschweig, 1997. MR:1757192 (2000m:11049)

[Se2] Serre, J.-P., Local fields, Springer-Verlag, New York-Berlin, 1979. MR0554237 (82e:12016)

[Shi] Shintani, T., On Dirichlet series whose coefficients are class numbers of integral binary cubic forms, J. Math. Soc. Japan 24 (1972), 132-188. MR0289428(44:6619)

[Si] Siegel, C. L., Lectures on the geometry of numbers, notes by B. Friedman, rewritten by K. Chandrasekharan with the assistance of R. Suter, Springer-Verlag, Berlin, 1989. MR1020761 (91d:11070)

[Sil] Silverman, J. H., Advanced topics in the arithmetic of elliptic curves, Springer-Verlag, New York, 1994. MR.1312368 (96b:11074)

[Sil2] Silverman, J. H., The arithmetic of elliptic curves, Springer-Verlag, New York, 1985. MR 0817210 (87g:11070)

[Sil3] Silverman, J. H., The difference between the Weil height and the canonical height on elliptic curves, Math. Comp. 55 (1990), 723-743. MR.1035944(91d:11063)

[Sil4] Silverman, J. H., Lower bound for the canonical height on elliptic curves, Duke Math. J. 48 (1981), no. 3, 633-648. MR0630588 (82k:14043)

[Sil5] Silverman, J. H., Lower bounds for height functions, Duke Math. J. 51 (1984), no. 2, 395-403. MR0747871 (87d:11039)

[Sil6] Silverman, J. H., A quantitative version of Siegel's theorem: integral points on elliptic curves and Catalan curves, J. Reine Angew. Math. 378 (1987), 60-100. MR0895285 (89g:11047) 
[So] Soundararajan, K., Divisibility of class numbers of imaginary quadratic fields, J. London Math. Soc. (2) 61 (2000), no. 3, 681-690. MR1766097 (2001i:11128)

[Wo] Wong, S., Automorphic forms on GL(2) and the rank of class groups, J. Reine Angew. Math. 515 (1999), 125-153. MR1717617 (2000g:11042)

[Wo2] Wong, S., On the rank of ideal class groups, Number theory (Ottawa, ON, 1996), 377383, CRM Proc. Lecture Notes, 19, Amer. Math. Soc., Providence, RI, 1999. MR1684617 (2000k:11126)

Department of Mathematics, Yale University, New Haven, Connecticut 06520

Current address: Département de mathématiques et de statistique, Université de Montréal, CP 6128 succ Centre-Ville, Montréal QC H3C 3J7, Canada

E-mail address: helfgott@dms.umontreal.ca

Department of Mathematics, Massachusetts institute of Technology, Cambridge, MASSACHUSETTS 02139-4307

Current address: Institute for Advanced Study, Einstein Drive, Princeton, NJ 08540

E-mail address: akshay@ias.edu 